IMA Journal of Numerical Analysis (2014) 34, 123-146

doi:10.1093/imanum/drt001

Advance Access publication on May 12, 2013

\title{
A priori error analysis for finite element approximations of the Stokes problem on dynamic meshes
}

\author{
ANDREAS BRENNER* AND EBERHARD BÄNSCH \\ Applied Mathematics III, University Erlangen-Nürnberg, Haberstraße 2, 91058 Erlangen, Germany \\ *Corresponding author: brenner@math.fau.de baensch@math.fau.de \\ AND \\ Markus Bause \\ Department of Mechanical Engineering, Helmut Schmidt University, Holstenhofweg 85, \\ 22043 Hamburg, Germany \\ bause@hsu-hh.de
}

[Received on 17 June 2011; revised on 20 December 2012]

\begin{abstract}
In this article we study finite element approximations of the time-dependent Stokes system on dynamically changing meshes. Applying the backward Euler method for time discretization we use the discrete Helmholtz or Stokes projection to evaluate the solution at time $t_{n-1}$ on the new spatial mesh at time $t_{n}$. The theoretical results consist of a priori error estimates that show a dependence on the time step size not better than $\mathcal{O}(1 / \Delta t)$. These surprisingly pessimistic upper bounds are complemented by numerical examples giving evidence for a negative convergence rate, at least for a large range of time step sizes, and in this sense backing our theory. These observations imply that using adaptive meshes for incompressible flow problems is delicate and requires further investigation.
\end{abstract}

Keywords: Stokes equations; dynamic finite element meshes; adaptivity.

\section{Introduction}

Many applications in fluid dynamics involve localized and time-dependent phenomena such as sharp moving fronts, interior or boundary layers, shocks and complicated structures, where important physical phenomena take place. Examples can be found in technical and environmental engineering or in the life sciences. The reliable and efficient numerical approximation of solutions to such problems is still a challenging task. To resolve the dynamics of complex nonstationary flows with high accuracy and efficiency, it is necessary to use dynamic meshes (that is, meshes changing in time) for the discretization in space; cf., for example, Hoffmann \& Johnson (2003) and Bänsch \& Schmidt (2003). Flow problems with free boundaries have also enjoyed increasing interest in industrial applications; cf., for example, Bänsch (2001), Cuvelier (1985) and Tobiska \& Matthies (2002). In the numerical simulation of flows with free boundaries the computational domain, as well as the spatial mesh, change in each time step and have to be recomputed.

In general, dynamically changing meshes require a projection of the discrete solution at the old time level onto a different spatial mesh at the new time step. Too-frequent mesh changes, especially when the mesh topology is also changed, may yield serious convergence problems. This was shown in Dupont (1982) for even the simple case of the heat equation in one dimension: changing the mesh in each time step by $h / 2$ to the left, and then in the next time step back to the right, resulted in a dissipation term that 


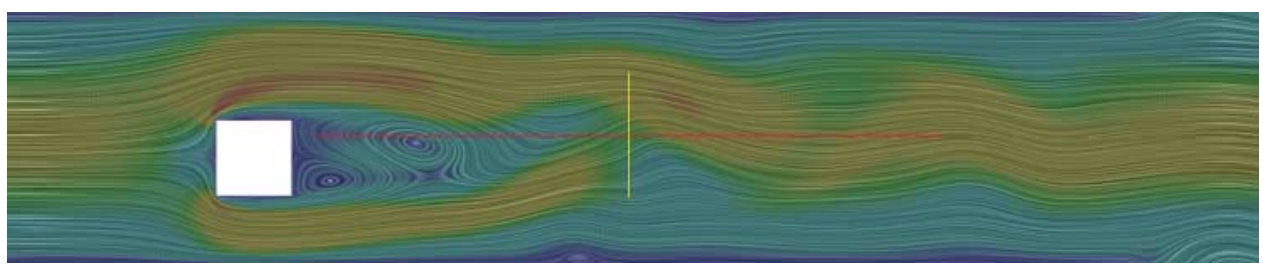

FIG. 1. von Karman vortex shedding. Line integral convolution visualization of the velocity for a certain time instant.

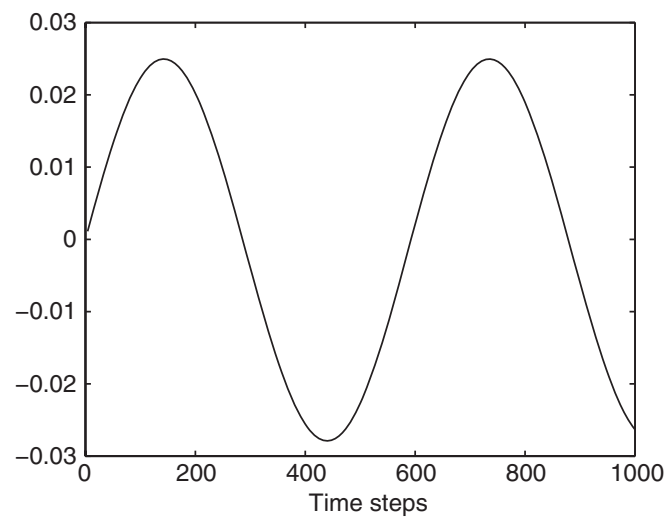

FIG. 2. Pressure versus time steps at a monitor point slightly behind the obstacle.

led to a completely wrong solution if $h^{4} / \Delta t \rightarrow \infty$. The reason for this is that the frequent and (for small time steps) rapid mesh changes introduce an artificial dissipation strong enough to damp the numerical solution to 0 , whereas the exact solution is nonvanishing.

The situation is even more delicate when an elliptic constraint is present. More specifically, in computing incompressible flows, the elliptic constraint of the solenoidal condition has to be taken into account. Numerical experiments have shown that depending on the specific projection this can cause additional and even stronger inaccuracies; cf., for example, Besier \& Wollner (2011) (in that paper, corresponding numerical results stimulate a theoretical investigation of using different projections). Note that this effect is different from the error mechanism for Dupont's example.

As an illustration, we computed a von Karman vortex shedding as depicted in Fig. 1. The flow is coming from the left given by a parabolic inflow profile, on the right outlet a do-nothing outflow condition is prescribed. The obstacle that gives rise to oscillatory flow behaviour for a medium Reynolds number is of a rectangular shape in order not to modify the geometry during mesh changes. The typical flow pattern is caught by probing the flow at a monitor point slightly behind the obstacle in Fig. 2. Figure 3 shows computations with a fixed fine mesh and for meshes that are randomly coarsened every 16 time steps (with simple interpolation of the old data onto the new mesh). Clearly, the pressure reacts in a very sensitive and instantaneous way to mesh changes, whereas the velocity behaves more reasonably for a long time. Thus, we deduce that the pressure is much more affected by mesh changes than the velocity, which is a phenomenon not present in, for instance, the heat equation. 

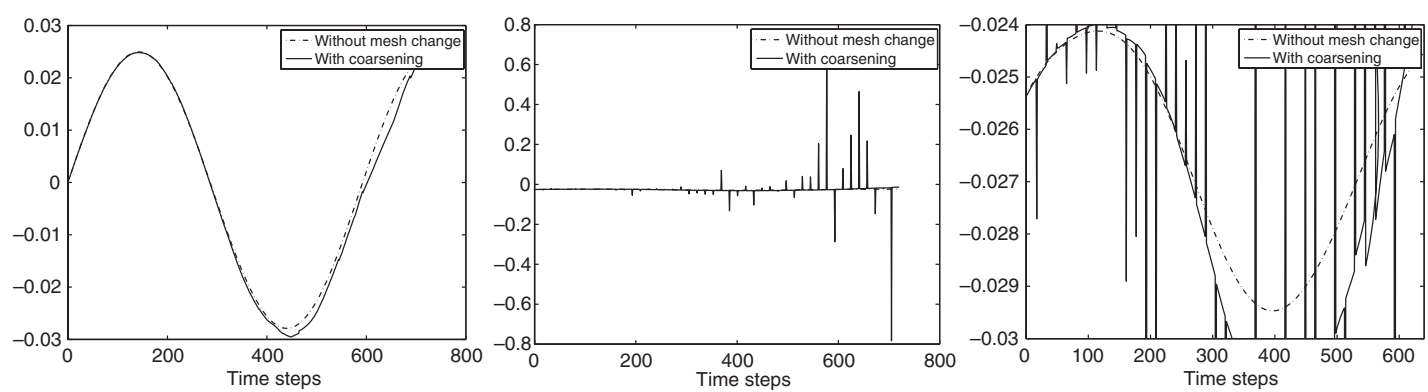

FIG. 3. $y$ component of the velocity (left), pressure (middle) and zoom into pressure plot (right), with and without mesh changes, at a monitor point slightly behind the obstacle.

In this paper we analyse theoretically and numerically finite element discretizations of nonstationary, incompressible flow problems on dynamic meshes. We will demonstrate that meshes varying in time can result in a loss of the order of convergence and even lead to a negative order of convergence of the discrete solution with respect to the time step size.

Despite its relevance, there are only a few articles dealing with the effect of mesh changes for parabolic problems. Let us mention the work of Dupont (1982), Eriksson \& Johnson (1991, 1995) and Yang (2000). In the pioneering work of Dupont (1982), for a one-dimensional parabolic model problem, it is shown that changing the mesh in a particular way in each time step leads to convergence of the numerical solution to a wrong answer. In Eriksson \& Johnson $(1991,1995)$ an a priori error analysis based on duality arguments is given for finite element approximations of a class of parabolic problems. Optimal-order error estimates are established under restrictions on the mesh changes, which have to satisfy either that the finite element spaces are embedded, $\mathcal{T}_{n} \subset \mathcal{T}_{n-1}$, or that the restriction $h_{n}^{2} \leqslant C \Delta t_{n}$ on the discretization step sizes is satisfied, where $h_{n}$ and $\Delta t_{n}$ denote the spatial and temporal step size, respectively, and $C$ is a constant. In Yang (2000) an error estimate for finite element approximations of a parabolic equation is established. There, the error is split into three parts, the temporal discretization error, the spatial discretization error and a projection error related to the number of mesh changes. However, no further estimate or convergence rate is given for the projection error. A naive estimate of this part leads to an upper bound depending reciprocally on the time step size.

Even less, however, is known for time-dependent problems with an elliptic constraint. As a prototype problem, in this paper we present a priori error estimates for finite element approximations with stable pairs of spaces (cf. Brezzi \& Fortin, 1982) on dynamic meshes for the Stokes problem

$$
\begin{aligned}
& \partial_{t} u-\Delta u+\nabla p=f, \quad \nabla \cdot u=0 \text { on }(0, T) \times \Omega, \\
& u=0 \text { on }(0, T) \times \partial \Omega,\left.\quad u\right|_{t=0}=u_{0} \text { on } \Omega .
\end{aligned}
$$

Here, $\Omega \subset \mathbb{R}^{d}$ with $d=2,3$ is a bounded polyhedral domain, $u$ denotes the velocity field, $p$ is the pressure, $f$ is a prescribed external force and $u_{0}$ denotes the prescribed initial velocity. Restricting our analysis to the linear Stokes problem modelling creeping flow, instead of considering the Navier-Stokes equations, is only done for the sake of simplicity. The effects of mesh changes are also observed for the Stokes system. The a priori error analysis of finite element approximations on dynamic meshes is of fundamental importance for ensuring their convergence as well as understanding and evaluating the asymptotic behaviour of discretizations. Studies of complex dynamic meshes, in particular those where the mesh topology is changing and the meshes are not obtained by refining or coarsening, are rare in 
the literature; cf., for example, Hoffmann \& Johnson (2003) and Dupont (1982). In our error analysis we follow the line of Yang (2000). We bound the approximation error by contributions that are due to the temporal discretization, the spatial discretization and the mesh changes. This separation of the error contributions allows us to study each source of inaccuracy separately. In contrast to Yang (2000), we derive convergence rates for the error caused by mesh changes. In our numerical experiments (see Section 5) we illustrate the sharpness of the given error estimates.

As mentioned above, in the numerical approximation of incompressible fluid flow the velocity approximation $U_{n-1}$ at time $t_{n-1}$ that has been computed on the mesh $\mathcal{T}_{n-1}$ has to be evaluated on the new spatial mesh $\mathcal{T}_{n}$ at time $t_{n}$. While $U_{n-1}$ satisfies a discrete solenoidal constraint with respect to the test space corresponding to mesh $\mathcal{T}_{n-1}$, this condition no longer holds on the new spatial mesh at time $t_{n}$, since the pair of finite element function spaces for velocity and pressure are strongly connected to the underlying mesh.

To get an idea of why this may lead to problems, let us consider an analogue of this situation for the semiimplicit Stokes equation, discretized in time by the backward Euler method,

$$
\frac{U_{n}-U_{n-1}}{\Delta t}-\Delta U_{n}+\nabla P_{n}=0
$$

Taking the divergence of this equation leads to

$$
\operatorname{div} \frac{U_{n}-U_{n-1}}{\Delta t}-\Delta \operatorname{div} U_{n}+\Delta P_{n}=0
$$

In the case that $U_{n}$ is divergence free, we get

$$
\Delta P_{n}=\frac{\operatorname{div} U_{n-1}}{\Delta t} .
$$

Now, if $U_{n-1}$ is not divergence free, then the Poisson pressure equation has an artificial right-hand side, scaled by $1 / \Delta t$.

Thus, in the spatial discrete case, the loss of the discrete divergence-free constraints leads to inaccuracies, in particular, in the pressure approximation. This problem was observed in Bänsch \& Schmidt (2003), where the authors propose a generalized Stokes projection of the old solution onto the new grid in order to cure spurious pressure peaks. In a recent work, Besier \& Wollner (2011) analyse this problem in more detail. In particular, Besier \& Wollner (2011) study the convergence behaviour of the solution for decreasing time step sizes under the rather strong assumption of only two mesh changes. The observation in Besier \& Wollner (2011) is that the standard $L^{2}$ projection of the velocity field at the old time onto the new spatial mesh $\mathcal{T}_{n-1}$ leads to diverging pressure approximations and, thereby, is not suitable for computations on dynamic meshes. Instead, the authors suggest and study the application of a divergence-free $L^{2}$ and a divergence-free $H_{0}^{1}$ projection.

In the present article we will show that neither the divergence-free $L^{2}$ nor the divergence-free $H_{0}^{1}$ projection are capable of handling dynamic finite element meshes. In particular, we give upper bounds for the error, which even suggest divergence of the discrete velocity and pressure, if the mesh is changed in each time step, as it might be necessary in applications of practical interest. This is done for two different test cases in Section 5.

At the current stage of our research we do not know how to eliminate the inaccuracies resulting from too-frequent mesh changes in time and how to project the velocity field onto the new spatial mesh in an optimal way without accumulating errors or unphysical oscillations in the variables close to the time of the mesh change. The intention of this work is to point out this source of trouble, to 
understand the phenomenon and to stimulate interest in analysing and solving the problem that is of practical relevance for all flow simulations on adaptive finite element meshes or with free boundaries and changing meshes. Moreover, to the best of our knowledge, an a priori error analysis for the finite element approximation of the Stokes problem on dynamically changing meshes has not been published before and may be of common interest.

Even though the Navier-Stokes equations are not explicitly studied here, similar observations will be made for Navier-Stokes flows. This was also pointed out in Besier \& Wollner (2011).

The rest of this paper is organized as follows. In Section 2 we introduce the finite element methods on dynamic meshes that are used here. In particular, two different methods for projecting the velocity at the old time onto the new spatial mesh are described. In Section 3 an a priori error analysis is given for the schemes introduced in Section 2. Error estimates including convergence rates for Stokes approximations on dynamic finite element meshes are proved. In Section 4 the projection error is studied in the case that only mesh refinements and coarsenings are permitted. In Section 5 the results of our numerical experiments are presented. They nicely illustrate the theoretical results and demonstrate the difficulties of accurate Stokes approximations on rapidly changing finite element meshes.

\section{Approximation schemes}

Let $\Omega \subset \mathbb{R}^{d}, d=2$ or $d=3$, be a bounded Lipschitz domain. For the sake of simplicity our notation does not differ between vector- and scalar-valued functions. As usual, $L^{2}(\Omega)$ denotes the space of functions defined and quadratically summable in $\Omega$ and $\|\cdot\|_{0}$ its norm. We denote the inner product in $L^{2}$ by $\langle\cdot, \cdot\rangle$. By $H^{m}(\Omega)=W^{m, 2}(\Omega)$, with $m \in \mathbb{N}_{0}$, we denote standard Sobolev spaces, equipped with the norm $\|u\|_{m}:=\left(\sum_{0 \leqslant|\alpha| \leqslant m}\left\|D^{\alpha} u\right\|^{2}\right)^{1 / 2}$ and $\|u\|:=\|u\|_{0} . H_{0}^{1}(\Omega)$ is the completion of $C_{0}^{\infty}(\Omega)$ in $H^{1}(\Omega)$, where $C_{0}^{\infty}(\Omega)$ is the space of all infinitely differentiable functions with compact support. Furthermore, let $J(\Omega)$ be the space of divergence-free functions given by

$$
J(\Omega):=\left\{\varphi \in H_{0}^{1}(\Omega) \mid \operatorname{div} \varphi=0\right\} .
$$

$H^{m}(\Omega) / \mathbb{R}$ denotes the quotient space of equivalence classes of elements of $H^{m}(\Omega)$ differing by constants. The norm in $H^{m}(\Omega) / \mathbb{R}$ is defined by $\|p\|_{H^{m} / \mathbb{R}}=\inf _{c \in \mathbb{R}}\|p+c\|_{m}$. For $m=0$ we set $L^{2} \backslash \mathbb{R}:=$ $H^{0} \backslash \mathbb{R}$. $L^{p}(0, T ; X)$, for any Banach space $\mathrm{X}$, is the space of measurable $X$-valued functions $u$ : $(0, T) \rightarrow X$ such that

$$
\begin{aligned}
\|u\|_{L^{p}(0, T ; X)} & :=\left(\int_{0}^{T}\|u(t)\|_{X}^{p} \mathrm{~d} t\right)^{1 / p}, \quad 1 \leqslant p<\infty, \\
\|u\|_{L^{\infty}(0, T ; X)} & :=\mathrm{ess} \sup _{0<t<T}\|u(t)\|_{X}, \quad p=\infty .
\end{aligned}
$$

The norm in $L^{p}(0, T ; X)$ is denoted by $\|\cdot\|_{L^{p}(X)}:=\|\cdot\|_{L^{p}(0, T ; X)}, 1 \leqslant p \leqslant \infty$. We will use $C$ as a generic constant, which may have different values in different occurrences.

With the above notation, the Stokes system (1.1) in weak form reads as follows: find $u \in L^{2}\left(0, T ; H_{0}^{1}(\Omega)\right), p \in L^{2}\left(0, T ; L^{2}(\Omega) / \mathbb{R}\right)$ with $\partial_{t} u \in L^{2}\left(0, T ; L^{2}(\Omega)\right)$ such that

$$
\begin{aligned}
\left\langle\partial_{t} u, v\right\rangle+\langle\nabla u, \nabla v\rangle-\langle p, \operatorname{div} v\rangle & =\langle f, v\rangle \quad \forall v \in H_{0}^{1}(\Omega), \\
\langle q, \operatorname{div} u\rangle & =0 \quad \forall q \in L^{2}(\Omega),
\end{aligned}
$$

for almost all $t \in(0, T)$, where $f \in L^{2}\left(0, T ; L^{2}(\Omega)\right)$. 
The divergence-free weak formulation used hereafter with the pressure variable eliminated is given as follows: find $u \in L^{2}(0, T ; J(\Omega))$ with $\partial_{t} u \in L^{2}\left(0, T ; L^{2}(\Omega)\right)$ such that

$$
\left\langle\partial_{t} u, v\right\rangle+\langle\nabla u, \nabla v\rangle=\langle f, v\rangle \quad \forall v \in J(\Omega),
$$

for almost all $t \in(0, T)$.

Clearly, (2.1) implies (2.2). Conversely, (2.2) implies the existence of a function $p$ satisfying (2.1) and $p$ can be recovered by

$$
\langle p, \operatorname{div} v\rangle=\left\langle\partial_{t} u, v\right\rangle+\langle\nabla u, \nabla v\rangle-\langle f, v\rangle \quad \forall v \in H_{0}^{1}(\Omega) \quad \text { and almost all } t \in(0, T) .
$$

Next, we introduce the discrete schemes that are studied in this work. For the temporal discretization of the Stokes system (1.1) we consider a mesh $\left\{t_{n}\right\}, n=0, \ldots, \mathcal{N}$, with $t_{0}=0$ and $t_{\mathcal{N}}=T$, for the time variable $t$ and define $\Delta t_{n}=t_{n}-t_{n-1}$. For $n=0,1, \ldots, N$, let $\mathcal{T}_{n}=\{K\}$ be a quasi-uniform conforming triangulation of mesh size $h_{n}, 0<h_{n}<1$, of the domain $\Omega$ at time $t_{n}$. The maximal mesh size is denoted by $h:=\max _{n} h_{n}$. The finite element spaces to be used for approximating the velocity space $H_{0}^{1}(\Omega)$ and the pressure space $L^{2}(\Omega)$ at time $t_{n}$ are of the form

$$
\begin{aligned}
& V_{n}=\left\{v_{n} \in H_{0}^{1}(\Omega) \mid v_{n \mid K} \in \mathbb{P}(K) \forall K \in \mathcal{T}_{n}\right\}, \\
& Q_{n}=\left\{q_{n} \in L^{2}(\Omega) \mid q_{n \mid K} \in \mathbb{Q}(K) \forall K \in \mathcal{T}_{n}\right\},
\end{aligned}
$$

where $\mathbb{P}(K)$ and $\mathbb{Q}(K)$ are spaces of polynomials on $K$ and $n=0,1, \ldots, N$.

We assume that the discrete spaces satisfy the following approximation properties: for each $u \in$ $H^{k+1}(\Omega) \cap H_{0}^{1}(\Omega)$ and $p \in H^{k}(\Omega)$ and for $n=0,1, \ldots, N$, there exist approximations $i_{n} u \in V_{n}$ and $j_{n} p \in Q_{n}$ such that

$$
\begin{aligned}
&\left\|u-i_{n} u\right\|+h_{n}\left\|\nabla\left(u-i_{n} u\right)\right\| \leqslant C h_{n}^{k+1}\|u\|_{k+1}, \\
&\left\|p-j_{n} p\right\|_{L^{2}(\Omega) / \mathbb{R}} \leqslant C h_{n}^{k}\|p\|_{H^{k}(\Omega) / \mathbb{R}} .
\end{aligned}
$$

The finite element space that is used for approximating the set $J(\Omega)$ of all divergence-free velocity fields at time $t_{n}$ is defined by

$$
J_{n}:=\left\{u_{n} \in V_{n} \mid\left\langle q_{n}, \operatorname{div} u_{n}\right\rangle=0 \forall q_{n} \in Q_{n}\right\} .
$$

We note that the finite element spaces $V_{n}, Q_{n}$ and $J_{n}$ no matching list are allowed to change in time. To ensure that the discrete pressure $p_{n} \in Q_{n}$ depends continuously on the discrete velocity $u_{n} \in J_{n}$ uniformly in $h_{n}$ for $n=0,1, \ldots, N$, we assume that the inf-sup condition holds.

For each $p_{n} \in Q_{n}$ there exists a nontrivial function $\varphi_{n} \in V_{n}$ such that

$$
\left|\left\langle p_{n}, \operatorname{div} \varphi_{n}\right\rangle\right| \geqslant C\left\|\nabla \varphi_{n}\right\|\left\|p_{n}\right\|_{L^{2} / \mathbb{R}}
$$

For the solution $u \in J(\Omega)$ and $p \in L^{2} / \mathbb{R}$ of (1.1) we define approximations $S_{n} u \in J_{n}$ satisfying

$$
\left\langle\nabla S_{n} u, \nabla v_{n}\right\rangle=\left\langle\nabla u, \nabla v_{n}\right\rangle-\left\langle p, \operatorname{div} v_{n}\right\rangle \quad \forall v_{n} \in J_{n} .
$$

We remark that $S_{n} u$ is vaguely defined since $S_{n}$ depends on the pressure as well. But for reasons of simplicity we follow the notation in Heywood \& Rannacher (1982, Section 4). 
Furthermore, we assume that the following error estimates are satisfied for $n=0,1, \ldots, N$ :

$$
\begin{gathered}
\left\|\left(I-S_{n}\right) u\right\|+h_{n}\left\|\nabla\left(\left(I-S_{n}\right) u\right)\right\| \leqslant C h_{n}^{k+1}\left(\|u\|_{k+1}+\|p\|_{H^{k} / \mathbb{R}}\right), \\
\left\|\left(I-S_{n}\right) \partial_{t} u\right\|+h_{n}\left\|\nabla\left(\left(I-S_{n}\right) \partial_{t} u\right)\right\| \leqslant C h_{n}^{k+1}\left(\left\|\partial_{t} u\right\|_{k+1}+\left\|\partial_{t} p\right\|_{H^{k} / \mathbb{R}}\right) .
\end{gathered}
$$

Supposing that the solution of the steady form of the Stokes system is sufficiently regular, these estimates are verified, for example, in Heywood \& Rannacher (1988).

Next, we define the $L^{2}$ or Helmholtz projection $\Pi_{n}: H_{0}^{1}(\Omega) \mapsto J_{n}$ by

$$
\left\langle\Pi_{n} u, v_{n}\right\rangle=\left\langle u, v_{n}\right\rangle \quad \forall v_{n} \in J_{n},
$$

and likewise the $H^{1}$ or Stokes projection $\Lambda_{n}: H_{0}^{1}(\Omega) \mapsto J_{n}$ by

$$
\left\langle\nabla \Lambda_{n} u, \nabla v_{n}\right\rangle=\left\langle\nabla u, \nabla v_{n}\right\rangle \quad \forall v_{n} \in J_{n} .
$$

REMARK 2.1 An equivalent formulation of the above definitions in terms of the mixed finite element pair $\left(V_{n}, Q_{n}\right)$ reads as follows: for $U \in V$ find a pair $(\hat{U}, \hat{P}) \in V_{n} \times Q_{n}$ such that

$$
\begin{aligned}
\left\langle\hat{U}-U, v_{n}\right\rangle+\left\langle\hat{P}, \operatorname{div} v_{n}\right\rangle=0 & \forall v_{n} \in V_{n}, \\
\left\langle q_{n}, \operatorname{div} \hat{U}\right\rangle=0 & \forall q_{n} \in Q_{n}
\end{aligned}
$$

for the Helmholtz projection and $q=1$ for the Stokes projection, respectively.

$$
\begin{aligned}
\left\langle\nabla(\hat{U}-U), \nabla v_{n}\right\rangle+\left\langle\hat{P}, \operatorname{div} v_{n}\right\rangle=0 & \forall v_{n} \in V_{n}, \\
\left\langle q_{n}, \operatorname{div} \hat{U}\right\rangle=0 & \forall q_{n} \in Q_{n} .
\end{aligned}
$$

Here, we use the backward Euler method for discretizing the divergence-free weak form (2.2) of the Stokes system (1.1) in time. Then, we consider the following fully discrete scheme for solving (1.1).

Algorithm 2.2 Suppose that $U_{0}$ is an initial approximation of $u(\cdot, 0)$. For $n=1, \ldots, N$, first compute the projection $\hat{U}_{n-1} \in J_{n}$ by a grid projection, either $\hat{U}_{n-1}=\Pi_{n} U_{n-1}$ or $\hat{U}_{n-1}=\Lambda_{n} U_{n-1}$, and then compute $U_{n} \in J_{n}$ by

$$
\left\langle\frac{U_{n}-\hat{U}_{n-1}}{\Delta t_{n}}, v_{n}\right\rangle+\left\langle\nabla U_{n}, \nabla v_{n}\right\rangle=\left\langle f_{n}, v_{n}\right\rangle \quad \forall v_{n} \in J_{n},
$$

as well as $P_{n} \in Q_{n}$ by

$$
\left\langle P_{n}, \operatorname{div} v_{n}\right\rangle=\left\langle\frac{U_{n}-\hat{U}_{n-1}}{\Delta t_{n}}, v_{n}\right\rangle+\left\langle\nabla U_{n}, \nabla v_{n}\right\rangle-\left\langle f_{n}, v_{n}\right\rangle \quad \forall v_{n} \in V_{n},
$$

where $f_{n}=f\left(t_{n}\right)$.

\section{Error estimates on dynamic meshes}

A lot of work has been done on the numerical analysis of finite element approximations of the Stokes or even Navier-Stokes equations; cf., for example, Heywood \& Rannacher (1982, 1990), Bause (2005), 
Girault \& Raviart (1987) and Temam (1983). Either energy techniques or functional analytical methods like semigroup theory have been applied. In particular, convergence rates have been proved. However, in all of these analyses, static finite element meshes have been assumed. As far as we know, error estimates for finite element approximations of the Stokes system on dynamic meshes have not been established yet. In this section we provide error estimates for the Stokes system on arbitrarily changing meshes. These estimates yield upper bounds. Assuming that the estimates are not too pessimistic they might give a better understanding of the effects of the divergence of finite element approximations on dynamic meshes in the case of decreasing time step sizes. In our numerical computations we have observed such cases of divergence. Examples illustrating this effect are given in Section 5.

For the sake of completeness and for comparison we shall recall some error estimates that were proved in He (2008) for finite element Navier-Stokes approximations on meshes not changing in time. If the Navier-Stokes equations are discretized in time by the backward Euler method and in space by a stable pair of finite element spaces for velocity and pressure of polynomial order $k$ and $k-1$, respectively, fulfilling (2.4), and if the mesh does not change in time, then for $n=1, \ldots, N$ it holds

$$
\left\|U_{n}-u\left(t_{n}\right)\right\| \leqslant C\left(\Delta t+h^{k+1}\right) \quad \text { and } \quad\left\|P_{n}-p\left(t_{n}\right)\right\| \leqslant C\left(\Delta t+h^{k}\right) .
$$

\subsection{A priori estimates on dynamic meshes by the Helmholtz projection}

First, we consider Algorithm 2.2 equipped with the Helmholtz projection (2.9) of the velocity field $U_{n-1}$ on the new spatial mesh $\mathcal{T}_{n}$. For this scheme the following error estimates can be established.

THEOREM 3.1 Suppose that $u$ and $p$ are sufficiently regular solutions of (2.2) and (2.3), respectively. For $n=1, \ldots, N$ let $U_{n}$ and $P_{n}$ be the solution of Algorithm 2.2 with grid projection (2.9). Then, the error estimates

$$
\begin{gathered}
\max _{1 \leqslant n \leqslant m}\left\|U_{n}-u_{n}\right\|^{2} \leqslant C\left(E_{0}+\max _{1 \leqslant n \leqslant m}\left\|\left(I-S_{n}\right) u_{n}\right\|^{2}\right), \\
\sum_{n=1}^{m} \Delta t_{n}\left\|\nabla\left(U_{n}-u_{n}\right)\right\|^{2} \leqslant C\left(E_{0}+\sum_{n=1}^{m} \Delta t_{n}\left\|\nabla\left(I-S_{n}\right) u_{n}\right\|^{2}\right), \\
\sum_{n=1}^{m} \Delta t_{n}\left\|P_{n}-p_{n}\right\|^{2} \leqslant C\left(E_{1}+\sum_{n=1}^{m} \Delta t_{n}\left\|\nabla\left(I-S_{n}\right) u_{n}\right\|^{2}\right)
\end{gathered}
$$

are satisfied for $1 \leqslant m \leqslant N$, where for $i=0,1$,

$$
\begin{aligned}
E_{i}= & \left\|U_{0}-u_{0}\right\|^{2}+\left[\sum_{n=1}^{m} \int_{t_{n-1}}^{t_{n}}\left\|\left(I-S_{n}\right) \partial_{t} u\right\| \mathrm{d} t\right]^{2} \\
& +\left[\sum_{n=1}^{m} \Delta t_{n} \int_{t_{n-1}}^{t_{n}}\left\|\partial_{t}^{2} u\right\| \mathrm{d} t\right]^{2}+\left[\sum_{n=1}^{m} h_{n}^{-i}\left\|\left(S_{n}-S_{n-1}\right) u_{n-1}\right\|\right]^{2} .
\end{aligned}
$$

Here, $S_{n}$ denotes the projection defined by $(2.7)$ and $\left(u_{n}, p_{n}\right):=\left(u\left(t_{n}\right), p\left(t_{n}\right)\right)$. 
A PRIORI ERROR ANALYSIS FOR FINITE ELEMENT APPROXIMATIONS

Proof. We put

$$
\begin{aligned}
& \theta_{n}=U_{n}-S_{n} u_{n}, \quad \hat{\theta}_{n-1}=\hat{U}_{n-1}-S_{n} u_{n-1}, \\
& \eta_{n}=u_{n}-S_{n} u_{n}, \quad \hat{\eta}_{n-1}=u_{n-1}-S_{n} u_{n-1},
\end{aligned}
$$

and define the time discretization error

$$
\rho_{n}:=\frac{u_{n}-u_{n-1}}{\Delta t_{n}}-\partial_{t} u_{n} .
$$

Subtracting (2.13) from (2.1) yields

$$
\left\langle\frac{U_{n}-\hat{U}_{n-1}}{\Delta t_{n}}-\frac{u_{n}-u_{n-1}}{\Delta t_{n}}, v_{n}\right\rangle+\left\langle\nabla\left(U_{n}-u_{n}\right), \nabla v_{n}\right\rangle+\left\langle p_{n}, \operatorname{div} v_{n}\right\rangle=-\left\langle\rho_{n}, v_{n}\right\rangle,
$$

where

$$
\left\|\rho_{n}\right\|=\left\|\frac{u_{n}-u_{n-1}}{\Delta t_{n}}-\partial_{t} u_{n}\right\| \leqslant \int_{t_{n-1}}^{t_{n}}\left\|\partial_{t}^{2} u\right\| \mathrm{d} t .
$$

Using (2.7), it follows that

$$
\left\langle\frac{\theta_{n}-\hat{\theta}_{n-1}}{\Delta t_{n}}, v_{n}\right\rangle+\left\langle\nabla \theta_{n}, \nabla v_{n}\right\rangle=\left\langle\frac{\eta_{n}-\hat{\eta}_{n-1}}{\Delta t_{n}}-\rho_{n}, v_{n}\right\rangle \quad \forall v_{n} \in J_{n} .
$$

The Helmholtz projection (2.9) satisfies

$$
\left\langle\hat{\theta}_{n-1}-\theta_{n-1}, v_{n}\right\rangle=\left\langle\hat{\eta}_{n-1}-\eta_{n-1}, v_{n}\right\rangle \quad \forall v_{n} \in J_{n} .
$$

Choosing $v_{n}=\theta_{n}$ in (3.6), with (3.7) and the identity

$$
\left\langle\theta_{n}-\theta_{n-1}, \theta_{n}\right\rangle=\frac{1}{2}\left(\left\|\theta_{n}\right\|^{2}-\left\|\theta_{n-1}\right\|^{2}+\left\|\theta_{n}-\theta_{n-1}\right\|^{2}\right),
$$

we obtain the error equation

$$
\frac{1}{2}\left\|\theta_{n}\right\|^{2}-\frac{1}{2}\left\|\theta_{n-1}\right\|^{2}+\Delta t_{n}\left\|\nabla \theta_{n}\right\|^{2} \leqslant\left\|\theta_{n}\right\|\left(\left\|\hat{\eta}_{n-1}-\eta_{n-1}\right\|+\left\|\eta_{n}-\hat{\eta}_{n-1}\right\|+\Delta t_{n}\left\|\rho_{n}\right\|\right) .
$$

Summing (3.8) from $n=1$ to $m$ with $1 \leqslant m \leqslant N$, we obtain

$$
\begin{aligned}
& \max _{1 \leqslant n \leqslant m}\left\|\theta_{n}\right\|^{2}-\left\|\theta_{0}\right\|^{2}+\sum_{n=1}^{m} \Delta t_{n}\left\|\nabla \theta_{n}\right\|^{2} \\
& \quad \leqslant 2 \sum_{n=1}^{m}\left\|\theta_{n}\right\|\left(\left\|\hat{\eta}_{n-1}-\eta_{n-1}\right\|+\left\|\eta_{n}-\hat{\eta}_{n-1}\right\|+\Delta t_{n}\left\|\rho_{n}\right\|\right) \\
& \quad \leqslant \frac{1}{2} \max _{1 \leqslant n \leqslant m}\left\|\theta_{n}\right\|^{2}+C\left(\left[\sum_{n=1}^{m}\left\|\hat{\eta}_{n-1}-\eta_{n-1}\right\|\right]^{2}+\left[\sum_{n=1}^{m}\left\|\eta_{n}-\hat{\eta}_{n-1}\right\|\right]^{2}+\left[\sum_{n=1}^{m} \Delta t_{n}\left\|\rho_{n}\right\|\right]^{2}\right) .
\end{aligned}
$$


For the interpolation error it holds

$$
\left[\sum_{n=1}^{m}\left\|\eta_{n}-\hat{\eta}_{n-1}\right\|\right]^{2} \leqslant C\left[\sum_{n=1}^{m} \int_{t_{n-1}}^{t_{n}}\left\|\left(I-S_{n}\right) \partial_{t} u\right\| \mathrm{d} t\right]^{2}
$$

Together with the identity $\left\|\hat{\eta}_{n-1}-\eta_{n-1}\right\|=\left\|\left(S_{n}-S_{n-1}\right) u_{n-1}\right\|$ this proves assertions (3.2) and (3.3). It remains to show (3.4). The inf-sup condition (2.6) yields

$$
\begin{aligned}
\left\|P_{n}-p_{n}\right\| & \leqslant\left\|p_{n}-j_{n} p_{n}\right\|+C \sup _{v_{n} \in V_{n}} \frac{\left\langle P_{n}-j_{n} p_{n}, \operatorname{div} v_{n}\right\rangle}{\left\|\nabla v_{n}\right\|} \\
& \leqslant(1+C)\left\|p_{n}-j_{n} p_{n}\right\|+C \sup _{v_{n} \in V_{n}} \frac{\left\langle P_{n}-p_{n}, \operatorname{div} v_{n}\right\rangle}{\left\|\nabla v_{n}\right\|},
\end{aligned}
$$

where $j_{n} p_{n}$ is the approximation of $p_{n}$ defined in (2.4). Subtracting (2.14) from (2.3), we get

$$
\left\langle P_{n}-p_{n}, \operatorname{div} v_{n}\right\rangle=\left\langle\frac{U_{n}-u_{n}-\left(\hat{U}_{n-1}-u_{n-1}\right)}{\Delta t_{n}}, v_{n}\right\rangle+\left\langle\nabla\left(U_{n}-u_{n}\right), \nabla v_{n}\right\rangle+\left\langle\rho_{n}, v_{n}\right\rangle .
$$

Substituting (3.11) into (3.10), we obtain

$$
\left\|P_{n}-p_{n}\right\| \leqslant C\left(\left\|p_{n}-j_{n} p_{n}\right\|+\frac{\left\|\theta_{n}-\hat{\theta}_{n-1}\right\|_{V_{n}^{*}}}{\Delta t_{n}}+\frac{\left\|\eta_{n}-\hat{\eta}_{n-1}\right\|_{V_{n}^{*}}}{\Delta t_{n}}+\left\|\nabla\left(U_{n}-u_{n}\right)\right\|+\left\|\rho_{n}\right\|\right),
$$

where $\|\cdot\|_{V_{n}^{*}}$ is the discrete dual norm

$$
\left\|w_{n}\right\|_{V_{n}^{*}}:=\sup _{v_{n} \in V_{n}, v_{n} \neq 0} \frac{\left\langle w_{n}, v_{n}\right\rangle}{\left\|\nabla v_{n}\right\|} .
$$

Squaring (3.12), multiplying by $\Delta t_{n}$ and summing from $n=1$ to $m$, it follows

$$
\begin{aligned}
\sum_{n=1}^{m} \Delta t_{n}\left\|P_{n}-p_{n}\right\|^{2} \leqslant & C\left(\sum_{n=1}^{m} \frac{\left\|\theta_{n}-\hat{\theta}_{n-1}\right\|_{V_{n}^{*}}^{2}}{\Delta t_{n}}+\sum_{n=1}^{m} \frac{\left\|\eta_{n}-\hat{\eta}_{n-1}\right\|_{V_{n}^{*}}^{2}}{\Delta t_{n}}\right. \\
& \left.+\sum_{n=1}^{m} \Delta t_{n}\left\|p_{n}-j_{n} p_{n}\right\|^{2}+\sum_{n=1}^{m} \Delta t_{n}\left\|\nabla\left(U_{n}-u_{n}\right)\right\|^{2}+\sum_{n=1}^{m} \Delta t_{n}\left\|\rho_{n}\right\|^{2}\right) .
\end{aligned}
$$

Note that

$$
\frac{\left\|\eta_{n}-\hat{\eta}_{n-1}\right\|_{V_{n}^{*}}^{2}}{\Delta t_{n}}=\frac{1}{\Delta t_{n}}\left\|\left(1-S_{n}\right) \int_{t_{n-1}}^{t_{n}} \partial_{t} u(t) \mathrm{d} t\right\|_{V_{n}^{*}}^{2} \leqslant C \Delta t_{n}\left\|\left(1-S_{n}\right) \partial_{t} u(t)\right\|^{2} .
$$

Owing to (2.14), (3.3), (3.5) and (3.14), it only remains to estimate the first term on the right-hand side of (3.13). 
Testing (3.6) by $v_{n}=\theta_{n}-\hat{\theta}_{n-1}$ yields, using the inverse estimate,

$$
\frac{1}{\Delta t_{n}}\left\|\theta_{n}-\hat{\theta}_{n-1}\right\|^{2} \leqslant\left(\frac{1}{\Delta t_{n}}\left\|\eta_{n}-\hat{\eta}_{n-1}\right\|+\left\|\rho_{n}\right\|\right)\left\|\theta_{n}-\hat{\theta}_{n-1}\right\|+C h_{n}^{-1}\left\|\nabla \theta_{n}\right\|\left\|\theta_{n}-\hat{\theta}_{n-1}\right\| .
$$

By applying Young's inequality and summing we obtain

$$
\sum_{n=1}^{m} \frac{\left\|\theta_{n}-\hat{\theta}_{n-1}\right\|^{2}}{\Delta t_{n}} \leqslant C\left(\sum_{n=1}^{m} \Delta t_{n} \frac{\left\|\eta_{n}-\hat{\eta}_{n-1}\right\|^{2}}{\Delta t_{n}}+\sum_{n=1}^{m} \Delta t_{n}\left\|\rho_{n}\right\|^{2}+\sum_{n=1}^{m} h_{n}^{-1} \Delta t_{n}\left\|\nabla \theta_{n}\right\|^{2}\right) .
$$

Combining this inequality with (3.5), (3.14) and (3.9) proves the assertion (3.4).

Inequalities (3.2-3.4) show that the discretization error of the velocity and pressure variable are governed by four quantities reflecting different sources of errors: the first of the contributions to the right-hand side terms in (3.2-3.4) measures the error of the approximation of the initial value $u_{0}$, the second and third term of the right-hand sides describe the error of the discretization in space and time, respectively, and the last part describes the projection error that is caused by the mesh changes.

The projection error is bounded from above by

$$
\begin{aligned}
{\left[\sum_{n=1}^{m}\left\|\left(S_{n}-S_{n-1}\right) u_{n-1}\right\|\right]^{2} } & =\left[\sum_{n=1}^{m}\left\|\left(S_{n}-I\right) u_{n-1}-\left(S_{n-1}-I\right) u_{n-1}\right\|\right]^{2} \\
& \leqslant\left[\sum_{n=1}^{m} \delta_{n-1}\left(\left\|\left(S_{n}-I\right) u_{n-1}\right\|+\left\|\left(S_{n-1}-I\right) u_{n-1}\right\|\right)\right]^{2},
\end{aligned}
$$

where $\delta_{n}=0$ if $\mathcal{T}_{n}=\mathcal{T}_{n-1}$ and $\delta_{n}=1$ otherwise. Applying (2.8), we obtain from (3.16) that

$$
\sum_{n=1}^{m}\left\|\left(S_{n}-S_{n-1}\right) u_{n-1}\right\| \leqslant \sum_{n=1}^{m} \delta_{n-1}\left(h_{n}^{k+1}+h_{n-1}^{k+1}\right) \leqslant C \cdot M h^{k+1} \leqslant C \frac{h^{k+1}}{\Delta t}
$$

where $M \leqslant N$ denotes the number of mesh changes, $h=\max _{0 \leqslant n \leqslant N} h_{n}$ and $\Delta t=\min _{1 \leqslant n \leqslant N} \Delta t_{n}$. In (3.17), each mesh change leads to an additional error part depending reciprocally on the time step size $\Delta t$. Of course, we do not know if this estimate is really sharp. It might be pessimistic. The sharpness of (3.17) will be studied further in Section 5 by numerical experiments.

Together, Theorem 3.1 and inequality (3.17) yield the following asymptotic error estimates for fully discrete Stokes approximations on dynamic finite element meshes if the Helmholtz projection (2.9) of the velocity field $U_{n-1}$ onto the new spatial mesh $\mathcal{T}_{n}$ is used. 
COROllary 3.2 Under the assumptions of Theorem 3.1 it holds

$$
\begin{aligned}
\max _{1 \leqslant n \leqslant m}\left\|U_{n}-u_{n}\right\|^{2} & =\mathcal{O}\left(\left\|U_{0}-u_{0}\right\|^{2}+\Delta t^{2}+h^{2(k+1)}+\frac{h^{2(k+1)}}{\Delta t^{2}}\right), \\
\sum_{n=1}^{m} \Delta t\left\|\nabla\left(U_{n}-u_{n}\right)\right\|^{2} & =\mathcal{O}\left(\left\|U_{0}-u_{0}\right\|^{2}+\Delta t^{2}+h^{2 k}+\frac{h^{2(k+1)}}{\Delta t^{2}}\right), \\
\sum_{n=1}^{m} \Delta t\left\|P_{n}-p_{n}\right\|^{2} & =\mathcal{O}\left(\left\|U_{0}-u_{0}\right\|^{2}+\Delta t^{2}+h^{2 k}+\frac{h^{2 k}}{\Delta t^{2}}\right),
\end{aligned}
$$

where $h=\max _{1 \leqslant n \leqslant m} h_{n}$.

REMARK 3.3 Note that the projection error (3.16) also occurs in the case of the heat equation, where the Stokes operator is replaced by the elliptic projection operator; cf. Yang (2000). However, because of the saddle point structure of the Stokes system, is more delicate.

\subsection{A priori estimates on dynamic meshes by the Stokes projection}

Next, we study Algorithm 2.2 equipped with the Stokes projection (2.10) of the velocity field $U_{n-1}$ onto the new spatial mesh $\mathcal{T}_{n}$.

Theorem 3.4 Suppose that $u$ and $p$ are sufficiently regular solutions of (2.2) and (2.3). For $n=1, \ldots, N$ let $U_{n}$ and $P_{n}$ be solutions of Algorithm 2.2 with grid projection (2.10). Then, the error estimates

$$
\begin{gathered}
\sum_{n=1}^{m} \Delta t_{n}\left\|U_{n}-u_{n}\right\|^{2} \leqslant C\left(H+\sum_{n=1}^{m} \Delta t_{n}\left\|\left(I-S_{n}\right) u_{n}\right\|^{2}\right), \\
\max _{1 \leqslant n \leqslant m}\left\|\nabla\left(U_{n}-u_{n}\right)\right\|^{2} \leqslant C\left(H+\max _{1 \leqslant n \leqslant m}\left\|\nabla\left(I-S_{n}\right) u_{n}\right\|^{2}\right), \\
\sum_{n=1}^{m} \Delta t_{n}\left\|P_{n}-p_{n}\right\|^{2} \leqslant C\left(H+\sum_{n=1}^{m} \Delta t_{n}\left\|\left(I-S_{n}\right) u_{n}\right\|^{2}\right)
\end{gathered}
$$

are satisfied for $1 \leqslant m \leqslant N$, where

$$
\begin{aligned}
H= & \left\|\nabla\left(U_{0}-u_{0}\right)\right\|^{2}+\sum_{n=1}^{m} \frac{1}{\Delta t_{n}}\left[\int_{t_{n-1}}^{t_{n}}\left\|\left(I-S_{n}\right) \partial_{t} u\right\| \mathrm{d} t\right]^{2} \\
& +\sum_{n=1}^{m} \Delta t_{n}\left[\int_{t_{n-1}}^{t_{n}}\left\|\partial_{t}^{2} u\right\| \mathrm{d} t\right]^{2}+\sum_{n=1}^{m} \frac{\left\|\nabla\left(S_{n}-S_{n-1}\right) u_{n-1}\right\|^{2}}{\Delta t_{n}} .
\end{aligned}
$$

Here, $S_{n}$ denotes the projection defined by $(2.7)$ and $\left(u_{n}, p_{n}\right):=\left(u\left(t_{n}\right), p\left(t_{n}\right)\right)$.

Proof. First, we prove the assertion (3.20). Similarly to the proof of Theorem 3.1 we put

$$
\begin{array}{ll}
\theta_{n}=U_{n}-S_{n} u_{n}, & \hat{\theta}_{n-1}=\hat{U}_{n-1}-S_{n} u_{n-1}, \\
\eta_{n}=u_{n}-S_{n} u_{n}, & \hat{\eta}_{n-1}=u_{n-1}-S_{n} u_{n-1} .
\end{array}
$$


Now we choose $v_{n}=\theta_{n}-\hat{\theta}_{n-1}$ in the error equation (3.6) and obtain by Young's inequality,

$$
\frac{\left\|\theta_{n}-\hat{\theta}_{n-1}\right\|^{2}}{\Delta t_{n}}+\left\|\nabla \theta_{n}\right\|^{2}-\left\|\nabla \hat{\theta}_{n-1}\right\|^{2} \leqslant 2 \Delta t_{n}\left\|\rho_{n}\right\|^{2}+2 \frac{\left\|\eta_{n}-\hat{\eta}_{n-1}\right\|^{2}}{\Delta t_{n}} .
$$

Next, we note that the Stokes projection (2.10) implies

$$
\left\langle\nabla \hat{\theta}_{n-1}-\nabla \theta_{n-1}, \nabla v_{n}\right\rangle=\left\langle\nabla \hat{\eta}_{n-1}-\nabla \eta_{n-1}, \nabla v_{n}\right\rangle \quad \forall v_{n} \in J_{n}
$$

Choosing $v_{n}=\hat{\theta}_{n-1}$ we obtain, by Young's inequality,

$$
\left\|\nabla \hat{\theta}_{n-1}\right\|^{2}-\left\|\nabla \theta_{n-1}\right\|^{2} \leqslant 2 \sqrt{2}\left\|\nabla \hat{\eta}_{n-1}-\nabla \eta_{n-1}\right\|\left\|\nabla \theta_{n-1}\right\|+4\left\|\nabla \hat{\eta}_{n-1}-\nabla \eta_{n-1}\right\|^{2} .
$$

Inserting (3.23) into (3.22) and summing the resulting inequality from $n=1$ to $m$ gives

$$
\begin{aligned}
& \sum_{n=1}^{m} \frac{\left\|\theta_{n}-\hat{\theta}_{n-1}\right\|^{2}}{\Delta t_{n}}+\max _{1 \leqslant n \leqslant m}\left\|\nabla \theta_{n}\right\|^{2} \\
& \leqslant\left\|\nabla \theta_{0}\right\|^{2}+2 \sqrt{2} \max _{0 \leqslant n \leqslant m}\left\|\nabla \theta_{n}\right\| \sum_{n=1}^{m}\left\|\nabla \hat{\eta}_{n-1}-\nabla \eta_{n-1}\right\| \\
& \quad+C \sum_{n=1}^{m}\left(\Delta t_{n}\left\|\rho_{n}\right\|^{2}+\frac{\left\|\eta_{n}-\hat{\eta}_{n-1}\right\|^{2}}{\Delta t_{n}}+\left\|\nabla \hat{\eta}_{n-1}-\nabla \eta_{n-1}\right\|^{2}\right) \\
& \leqslant\left\|\nabla \theta_{0}\right\|^{2}+\frac{1}{2} \max _{0 \leqslant n \leqslant m}\left\|\nabla \theta_{n}\right\|^{2}+C\left(\left[\sum_{n=1}^{m}\left\|\nabla \hat{\eta}_{n-1}-\nabla \eta_{n-1}\right\|\right]^{2}\right. \\
& \left.\quad+\sum_{n=1}^{m}\left(\Delta t_{n}\left\|\rho_{n}\right\|^{2}+\frac{\left\|\eta_{n}-\hat{\eta}_{n-1}\right\|^{2}}{\Delta t_{n}}+\left\|\nabla \hat{\eta}_{n-1}-\nabla \eta_{n-1}\right\|^{2}\right)\right) .
\end{aligned}
$$

Therefore, it follows that

$$
\begin{aligned}
& \sum_{n=1}^{m} \frac{\left\|\theta_{n}-\hat{\theta}_{n-1}\right\|^{2}}{\Delta t_{n}}+\max _{1 \leqslant n \leqslant m}\left\|\nabla \theta_{n}\right\|^{2} \\
& \quad \leqslant C\left(\left\|\nabla \theta_{0}\right\|^{2}+\sum_{n=1}^{m} \Delta t_{n}\left\|\rho_{n}\right\|^{2}+\sum_{n=1}^{m} \frac{\left\|\eta_{n}-\hat{\eta}_{n-1}\right\|^{2}}{\Delta t_{n}}+\sum_{n=1}^{m} \frac{\left\|\nabla \hat{\eta}_{n-1}-\nabla \eta_{n-1}\right\|^{2}}{\Delta t_{n}}\right) .
\end{aligned}
$$

Finally, Eq. (3.20) is proved by applying the triangle inequality to $\left\|\nabla\left(U_{n}-u_{n}\right)\right\|$ and using the estimate (3.24).

To show the error estimate (3.19), we multiply (3.6) by $\Delta t_{n}^{2}$ and choose $v_{n}=\theta_{n}$. Then, we obtain that

$$
\frac{\Delta t_{n}}{2}\left(\left\|\theta_{n}\right\|^{2}-\left\|\hat{\theta}_{n-1}\right\|^{2}+\left\|\theta_{n}-\hat{\theta}_{n-1}\right\|^{2}\right)+\Delta t_{n}^{2}\left\|\nabla \theta_{n}\right\|^{2} \leqslant \Delta t_{n}^{2}\left\|\rho_{n}\right\|\left\|\theta_{n}\right\|+\Delta t_{n}\left\|\eta_{n}-\hat{\eta}_{n-1}\right\|\left\|\theta_{n}\right\| .
$$


Next, in (3.25) we shall bound the term $\left\|\hat{\theta}_{n-1}\right\|$ from above. By the Poincaré inequality together with inequality (3.23) we get

$$
\left\|\hat{\theta}_{n-1}\right\|^{2} \leqslant C\left\|\nabla \hat{\theta}_{n-1}\right\|^{2} \leqslant C\left(\left\|\nabla \theta_{n-1}\right\|^{2}+\left\|\nabla \hat{\eta}_{n-1}-\nabla \eta_{n-1}\right\|^{2}\right)
$$

Combining (3.25) and (3.26) and summing the resulting relation from $n=1$ to $m$ yields

$$
\begin{aligned}
\sum_{n=1}^{m} \Delta t_{n}\left\|\theta_{n}\right\|^{2} \leqslant & C\left(\sum_{n=1}^{m} \Delta t_{n}^{3}\left\|\rho_{n}\right\|^{2}+\sum_{n=1}^{m} \Delta t_{n}\left\|\eta_{n}-\hat{\eta}_{n-1}\right\|^{2}\right. \\
& \left.+\sum_{n=1}^{m} \Delta t_{n}\left\|\nabla \theta_{n-1}\right\|^{2}+\sum_{n=1}^{m} \Delta t_{n}\left\|\nabla \hat{\eta}_{n-1}-\nabla \eta_{n-1}\right\|^{2}\right)
\end{aligned}
$$

where the third term on the right-hand side of (3.27) can be further estimated by means of

$$
\sum_{n=1}^{m} \Delta t_{n}\left\|\nabla \theta_{n-1}\right\|^{2} \leqslant \max _{1 \leqslant n \leqslant m}\left\|\nabla \theta_{n-1}\right\|^{2} \sum_{n=1}^{m} \Delta t_{n} \leqslant T \max _{0 \leqslant n \leqslant m}\left\|\nabla \theta_{n}\right\|^{2} .
$$

Combined with (3.24), this proves the assertion (3.19).

The proof of the error estimate (3.21) for the pressure variable directly follows from inequalities (3.13) and (3.24).

Similarly to the case of the Helmholtz projection of $U_{n-1}$ on $\mathcal{T}_{n}$ considered above, the upper bounds given in Theorem 3.4 for the Stokes projection are governed by four quantities again, which measure the different sources of errors. Estimating the error caused by the mesh changes as in (3.16) and (3.17), it holds that

$$
\sum_{n=1}^{m}\left\|\nabla\left(S_{n}-S_{n-1}\right) u_{n-1}\right\| \leqslant C \frac{h^{k}}{\Delta t}
$$

where $h=\max _{n} h_{n}$ and $\Delta t=\min _{n} \Delta t_{n}$. Of course, the upper bound in (3.28) might be pessimistic again.

Together, Theorem 3.4 and inequality (3.28) yield the following asymptotic error estimates for fully discrete Stokes approximations on dynamic finite element meshes if the Stokes projection (2.10) of the velocity field $U_{n-1}$ onto the new spatial mesh $\mathcal{T}_{n}$ is used and constant time steps $\Delta t_{n}$ are assumed.

Corollary 3.5 Under the assumptions of Theorem 3.4 it holds that

$$
\begin{aligned}
\sum_{n=1}^{m} \Delta t\left\|U_{n}-u_{n}\right\|^{2} & =\mathcal{O}\left(\left\|\nabla\left(U_{0}-u_{0}\right)\right\|^{2}+\Delta t^{2}+h^{2(k+1)}+\frac{h^{2 k}}{\Delta t^{2}}\right), \\
\max _{1 \leqslant n \leqslant m}\left\|\nabla\left(U_{n}-u_{n}\right)\right\|^{2} & =\mathcal{O}\left(\left\|\nabla\left(U_{0}-u_{0}\right)\right\|^{2}+\Delta t^{2}+h^{2 k}+\frac{h^{2 k}}{\Delta t^{2}}\right), \\
\sum_{n=1}^{m} \Delta t\left\|P_{n}-p_{n}\right\|^{2} & =\mathcal{O}\left(\left\|\nabla\left(U_{0}-u_{0}\right)\right\|^{2}+\Delta t^{2}+h^{2 k}+\frac{h^{2 k}}{\Delta t^{2}}\right),
\end{aligned}
$$

where $h=\max _{1 \leqslant n \leqslant m} h_{n}$. 
We shall now compare the error estimates given in Theorem 3.1 for the Helmholtz projection (2.9) of the velocity field $U_{n-1}$ onto the new spatial mesh $\mathcal{T}_{n}$ with the error estimates given in Theorem 3.4 for the Stokes projection (2.10) of $U_{n-1}$. For this we introduce the discrete norms

$$
\begin{aligned}
\|v\|_{\ell^{2}\left(L^{2}\right)} & :=\|v\|_{\ell^{2}\left(0, T ; L^{2}(\Omega)\right)}:=\left(\sum_{1 \leqslant n \leqslant m} \Delta t_{n}\left\|v\left(\cdot, t_{n}\right)\right\|_{L^{2}(\Omega)}^{2}\right)^{1 / 2}, \\
\|v\|_{\ell^{\infty}\left(L^{2}\right)} & :=\|v\|_{\ell^{\infty}\left(0, T ; L^{2}(\Omega)\right)}:=\max _{1 \leqslant n \leqslant m}\left\|v\left(\cdot, t_{n}\right)\right\|_{L^{2}(\Omega)} .
\end{aligned}
$$

According to (3.17) and (3.28), an estimate of the contributions to the error bounds in Theorems 3.1 and 3.4 , respectively, that is, due to the projection of $U_{n-1}$ on $\mathcal{T}_{n}$ is given by

$$
\sum_{n=1}^{m}\left|\left(S_{n}-S_{n-1}\right) u_{n-1}\right|_{q} \leqslant C h^{k+1-q},
$$

where $q=1$ for the Stokes projection (2.10) and $q=0$ for the Helmholtz projection (2.9) with $h=\max _{1 \leqslant n \leqslant N} h_{n}$. Of course, the estimate (3.30) might be pessimistic. This will be studied further in Section 5. Combined with (3.30), Theorems 3.1 and 3.4 imply the following convergence rates for fully discrete Stokes approximations on finite element meshes varying in time: for the velocity field it holds that

$$
\begin{aligned}
\text { Helmholtz projection: } \quad\|U-u\|_{\ell^{\infty}\left(L^{2}\right)}=\mathcal{O}\left(\left\|U_{0}-u_{0}\right\|+\Delta t+h^{k+1}+\frac{h^{k+1}}{\Delta t}\right), \\
\text { Stokes projection: }\|U-u\|_{\ell^{2}\left(L^{2}\right)}=\mathcal{O}\left(\left\|\nabla\left(U_{0}-u_{0}\right)\right\|+\Delta t+h^{k+1}+\frac{h^{k}}{\Delta t}\right),
\end{aligned}
$$

and for the pressure variable we have that

$$
\begin{array}{cl}
\text { Helmholtz projection: } & \|P-p\|_{\ell^{2}\left(L^{2}\right)}=\mathcal{O}\left(\left\|U_{0}-u_{0}\right\|+\Delta t+h^{k}+\frac{h^{k}}{\Delta t}\right), \\
\text { Stokes projection: } & \|P-p\|_{\ell^{2}\left(L^{2}\right)}=\mathcal{O}\left(\left\|\nabla\left(U_{0}-u_{0}\right)\right\|+\Delta t+h^{k}+\frac{h^{k}}{\Delta t}\right) .
\end{array}
$$

First, we note that our upper error bounds for Stokes approximations on dynamic finite element meshes depend reciprocally on the time step size which implies a restriction of the mesh size $h$ with respect to the magnitude of the temporal step size $\Delta t$ in order to ensure convergence for vanishing mesh sizes in time and space. On static meshes the error estimates given in (3.1) yield convergence without such a smallness condition for $h$. Furthermore, we observe that in the approximation of the velocity field, one order of convergence gets lost with respect to the discretization in space if the Stokes projection instead of the Helmholtz projection of $U_{n-1}$ onto the new spatial mesh $\mathcal{T}_{n}$ is used. Moreover, the error estimate holds in the weaker $\|\cdot\|_{\ell^{2}\left(L^{2}\right)}$ norm. We note that the reciprocal dependence on $\Delta t$ arises only in the part of the upper bound originating from mesh changes. Our numerical calculations in Section 5 will show that both variants of projecting $U_{n-1}$ onto the new spatial mesh $\mathcal{T}_{n}$ are still unsatisfactory and can lead to divergence of the fully discrete unknowns if completely uncontrolled mesh changes are performed. 


\section{Projection error for mesh refining and coarsening}

In this section we study the error behaviour of finite element Stokes approximations in the case that only mesh refinements and coarsenings are permitted. In contrast to Section 3 we thus no longer allow arbitrary mesh changes. By restricting ourselves to refinement and coarsening steps we mimic mesh changes that could be based on adaptivity. This is the most popular application of dynamic mesh changes in practice. We will prove error bounds that no longer depend reciprocally on the time step size and hold uniformly with respect to the time step size, similarly to the case of static finite element meshes.

In (3.17) and (3.30), the worst case estimate

$$
\sum_{n=1}^{m}\left|\left(S_{n}-S_{n-1}\right) u_{n-1}\right|_{q} \leqslant C \frac{h^{k+1-q}}{\Delta t}
$$

was given for the error parts of Stokes approximations that are due to the changes, where $q=0$ if the Helmholtz projection (2.9) is used and $q=1$ if the Stokes projection (2.10) is used. If we restrict the mesh changes to pure refinement steps, then the following sharper estimate of the error parts (cf. Theorems 3.1 and 3.4) reflecting the mesh changes can be established.

THEOREM 4.1 Suppose that $u$ and $p$ are sufficiently regular solutions of (2.2) and (2.3). Let $h_{0}$ denote the finite element mesh size of the initial grid $\mathcal{T}_{0}$. Suppose that the initial grid is at most $Q$ times, $Q \leqslant m$, uniformly refined with mesh sizes $h_{i}=\frac{1}{2} h_{i-1}, 1 \leqslant i \leqslant Q$. Then, for the error part that is due to the mesh changes (cf. Theorems 3.1 and 3.4), it holds that

$$
\sum_{n=1}^{m}\left|\left(S_{n}-S_{n-1}\right) u_{n-1}\right|_{q} \leqslant C h_{0}^{k+1-q}\left(\|u\|_{L^{\infty}\left([0, T] ; H^{k+1-q}\right)}+\|p\|_{L^{\infty}\left([0, T] ; H^{k-q} \backslash \mathbb{R}\right)}\right),
$$

where $q=0$ for the Helmholtz projection and $q=1$ for the Stokes projection. The constant $C$ does not depend on the number of refinements.

Proof. Using (2.8), (3.16) and (3.17), we obtain that

$$
\sum_{n=1}^{m}\left|\left(S_{n}-S_{n-1}\right) u_{n-1}\right|_{q} \leqslant C \sum_{i=1}^{Q}\left(h_{i}^{k+1-q}-h_{i-1}^{k+1-q}\right) A_{i-1},
$$

where $A_{i}=\left\|u\left(t_{i}\right)\right\|_{k+1-q}+\left\|p\left(t_{i}\right)\right\|_{H^{k-q} \backslash \mathbb{R}}$ and $i \in I=\left\{n=1, \ldots, m \mid \mathcal{T}_{n} \neq \mathcal{T}_{n-1}\right\}$. For regular mesh refinements with $h_{i}=\frac{1}{2} h_{i-1}$ and, therefore, $h_{i}=h_{0} / 2^{-i}$ it follows that

$$
\begin{aligned}
\sum_{n=1}^{m}\left|\left(S_{n}-S_{n-1}\right) u_{n-1}\right|_{q} & \leqslant C h_{0}^{k+1-q}\|A\|_{L^{\infty}([0, T])} \sum_{i=1}^{Q} 2^{-i(k+1-q)}+2^{-(i-1)(k+1-q)} \\
& \leqslant C h_{0}^{k+1-q}\|A\|_{L^{\infty}([0, T])} \sum_{i=0}^{Q} 2^{-i(k+1-q)}
\end{aligned}
$$

Since the sum on the right-hand side is bounded from above independently of $Q$, assertion (4.1) is proved. 
REMARK 4.2 If pure mesh coarsenings are permitted, we get analogously that

$$
\sum_{n=1}^{m}\left|\left(S_{n}-S_{n-1}\right) u_{n-1}\right|_{q} \leqslant C h_{Q}^{k+1-q}\left(\|u\|_{L^{\infty}\left([0, T] ; H^{k+1-q}\right)}+\|p\|_{L^{\infty}\left([0, T] ; H^{k-q} \backslash \mathbb{R}\right)}\right),
$$

where $h_{Q}$ denotes the finite element mesh size of the coarsest grid.

Together, inequalities (4.1) and (4.2) indicate that mesh changes based on a refining and coarsening strategy of adaptivity should not lead to diverging approximations due to the performed mesh changes. This conclusion is in accordance with the observations and results that are presented in Besier \& Wollner (2011).

REMARK 4.3 Karakashian \& Makridakis (2004) study space-time continuous Galerkin methods with mesh modification in time for semilinear second-order hyperbolic equations. They show that mesh modifications lead to additional terms, known as 'jump terms', in the a priori error analysis if no further restrictions on the mesh changes are made. Precisely, the jump terms are the elliptic projection of the solution and its time derivative at times $t_{n}$ where mesh changes occur. These terms, albeit small, might cause the divergence of the numerical method. Thus, the structure of the a priori error estimate that is given in Karakashian \& Makridakis (2004) for hyperbolic problems is in good agreement with our error estimates for Stokes approximations given Theorems 3.1 and 3.4. Karakashian and Makridakis further show that the jump terms can be eliminated under the 'reasonable' assumption that all meshes contain a reference mesh $\mathcal{T}_{h}$. In such cases, convergence rates of optimal order can be guaranteed. Finally, they prove that an increased accuracy is obtained for the jump terms if the meshes differ only in regions of small area. In the two-dimensional case this region has to be an area of size $\mathcal{O}\left(h^{2}\right)$. Under this assumption about the meshes, optimal convergence rates are established again for the second-order hyperbolic model problem. Such restrictions on the mesh changes are not studied here because our interest is to show theoretically and numerically that arbitrary mesh changes can lead to divergence of Stokes approximations, even for divergence-free projections onto the modified meshes, and to stimulate interest in analysing this phenomenon.

\section{Numerical studies}

In this section we study numerically the behaviour of finite element approximations of the nonstationary Stokes equations on meshes varying in time. We show that changing the mesh in an 'uncontrolled way' between two time steps can cause a negative order of convergence of the discrete solution. Moreover, the numerical calculations illustrate the a priori convergence rates that were proved in Section 3 and characterize to what extent they are sharp. Numerical calculations are presented for both the Helmholtz and the Stokes projection.

In Besier \& Wollner (2011) it is shown that a small number of mesh changes (in their case one mesh change) could be tolerated without significant loss in accuracy if the Helmholtz or the Stokes projection of the velocity approximation is used instead of its $L^{2}$ projection for evaluating the discrete velocity on the new spatial mesh. Here, we will show that this observation does not hold true if the mesh varies in each time step as might arise in complex applications with sharp moving fronts or free boundaries. In Fig. 4 we demonstrate that the pressure error of a single mesh change will be smoothed, but if we change the meshes several times, the error blows up.

EXAMPLE 5.1 In our first example we study the convergence rates of the finite element approximation of the nonstationary Stokes problem. We consider problem (1.1) with $\Omega=(0,1)^{2}$ 

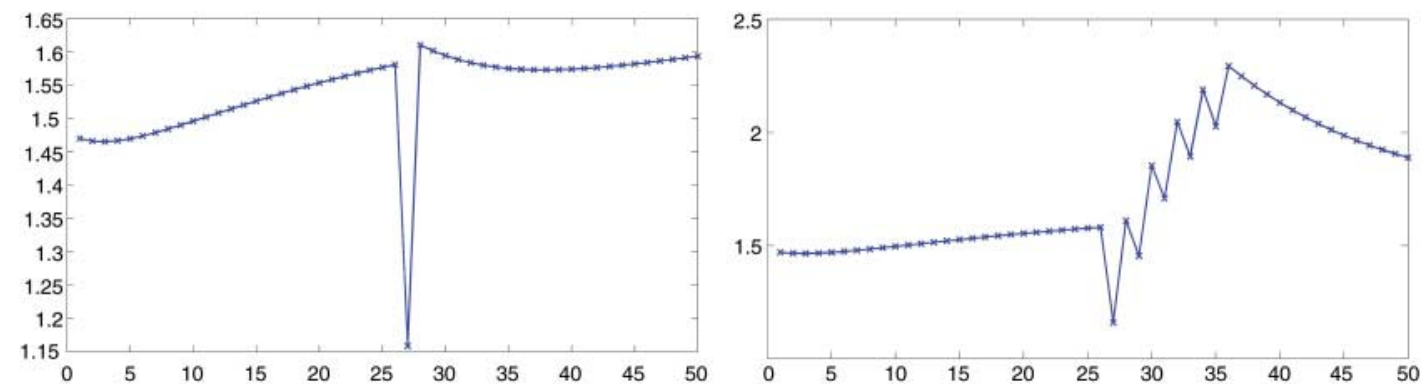

FIG. 4. Plots of time versus pressure error $\left\|P_{n}-p_{n}\right\|$ for Example 5.1 with time step $\Delta t=0.0001$. On the left-hand side two mesh changes, on the right-hand side ten mesh changes between triangulations with grid parameter $h=0.2$; cf. Fig. 5 .
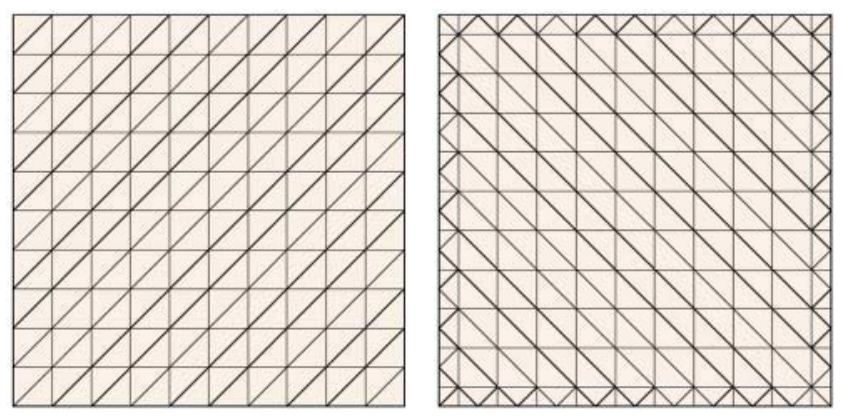

FIG. 5. Iterating triangulation in $(0,1)^{2}$ with grid parameter $h=0.1$.

on the time interval [0,0.01]. The analytical solution is given by $u\left(x_{1}, x_{2}\right)=\left(\frac{1}{2} \sin \left(4 \pi x_{1}\right)\right.$ $\left.\cos \left(2 \pi x_{2}\right), \sin \left(2 \pi x_{2}\right)\left(2 \sin ^{2}\left(2 \pi x_{1}\right)-1\right)\right)^{\mathrm{T}}$ and $p\left(x_{1}, x_{2}\right)=\frac{5}{2} \cos \left(4 \pi x_{1}\right) \cos \left(2 \pi x_{2}\right)$ with a corresponding right-hand-side term $f$. On the boundary $\partial \Omega$ we impose the Dirichlet data given by the analytical solution. The numerical solution is calculated by NAVIER (Bänsch, 1998) using $\mathcal{P}_{2} / \mathcal{P}_{1}$-Taylor-Hood elements. Since it is impossible to computationally realize arbitrary mesh changes without committing variational crimes we use meshes that are switched every time step between the triangulations shown in Fig. 5.

In Table 1 we summarize the calculated errors of velocity, velocity gradient and pressure for the Helmholtz projection for grid parameter $h=0.05$. We observe that under uniform refinement of the temporal discretization the errors increase as claimed in Theorem 3.1 and Corollary 3.2. The (divergence) rates given there are slightly more pessimistic compared with the numerical results that give a rate between $1 / \sqrt{\Delta t}$ and $1 / \Delta t$.

The results based on using the Stokes projection are summarized in Table 2 for grid parameter $h=0.05$. Similarly to the case of the Helmholtz projection we observe divergence of the solution for decreasing time step sizes, as predicted by Theorem 3.4 and Corollary 3.5. Again, the numerical results are slightly less pessimistic, yielding rates between $1 / \sqrt{\Delta t}$ and $1 / \Delta t$.

To compare the Helmholtz with the Stokes projection in Fig. 6, the velocity error $\|U-u\|_{\ell^{\infty}\left(L^{2}\right)}$ and the pressure error $\|P-P\|_{\ell^{2}\left(L^{2}\right)}$ are plotted for the grid parameter $h=0.05$. We realize that the Stokes projection is more sensitive concerning the predicted convergence rate than the Helmholtz projection. 
TABLE 1 Time step, error of velocity, velocity gradient and pressure with respective experimental order of convergence for the Helmholtz projection with grid parameter $h=0.05$

\begin{tabular}{lcccccccccc}
\hline$\Delta t$ & $\|U-u\|_{\ell^{\infty}\left(L^{2}\right)}$ & \multicolumn{2}{c}{$\|U-u\|_{\ell^{2}\left(L^{2}\right)}$} & \multicolumn{1}{c}{$\|\nabla(U-u)\|_{\ell^{\infty}\left(L^{2}\right)}$} & \multicolumn{1}{c}{$\|\nabla(U-u)\|_{\ell^{2}\left(L^{2}\right)}$} & $\|P-p\|_{\ell^{2}\left(L^{2}\right)}$ \\
\hline $2.00 \mathrm{e}-3$ & $3.13 \mathrm{e}-4$ & - & $2.90 \mathrm{e}-5$ & - & $4.00 \mathrm{e}-2$ & - & $3.73 \mathrm{e}-3$ & - & $1.63 \mathrm{e}-2$ & - \\
$1.00 \mathrm{e}-3$ & $3.16 \mathrm{e}-4$ & -0.01 & $2.93 \mathrm{e}-5$ & -0.01 & $3.94 \mathrm{e}-2$ & 0.02 & $3.73 \mathrm{e}-3$ & 0.00 & $1.61 \mathrm{e}-2$ & 0.02 \\
$5.00 \mathrm{e}-4$ & $3.17 \mathrm{e}-4$ & -0.00 & $2.93 \mathrm{e}-5$ & -0.00 & $3.88 \mathrm{e}-2$ & 0.03 & $3.67 \mathrm{e}-3$ & 0.02 & $1.59 \mathrm{e}-2$ & 0.02 \\
$2.50 \mathrm{e}-4$ & $3.21 \mathrm{e}-4$ & -0.02 & $3.00 \mathrm{e}-5$ & -0.03 & $3.88 \mathrm{e}-2$ & -0.00 & $3.67 \mathrm{e}-3$ & -0.00 & $1.58 \mathrm{e}-2$ & 0.01 \\
$1.25 \mathrm{e}-4$ & $3.53 \mathrm{e}-4$ & -0.14 & $3.28 \mathrm{e}-5$ & -0.13 & $4.18 \mathrm{e}-2$ & -0.11 & $3.88 \mathrm{e}-3$ & -0.08 & $1.52 \mathrm{e}-2$ & 0.05 \\
$6.25 \mathrm{e}-5$ & $4.49 \mathrm{e}-4$ & -0.35 & $4.03 \mathrm{e}-5$ & -0.30 & $5.05 \mathrm{e}-2$ & -0.27 & $4.48 \mathrm{e}-3$ & -0.21 & $1.50 \mathrm{e}-2$ & 0.03 \\
$3.13 \mathrm{e}-5$ & $6.56 \mathrm{e}-4$ & -0.55 & $5.63 \mathrm{e}-5$ & -0.48 & $6.41 \mathrm{e}-2$ & -0.34 & $5.53 \mathrm{e}-3$ & -0.30 & $1.52 \mathrm{e}-2$ & -0.03 \\
$1.56 \mathrm{e}-5$ & $1.07 \mathrm{e}-3$ & -0.71 & $8.83 \mathrm{e}-5$ & -0.65 & $7.86 \mathrm{e}-2$ & -0.29 & $7.04 \mathrm{e}-3$ & -0.35 & $1.54 \mathrm{e}-2$ & -0.02 \\
$7.81 \mathrm{e}-6$ & $1.89 \mathrm{e}-3$ & -0.82 & $1.52 \mathrm{e}-4$ & -0.78 & $9.75 \mathrm{e}-2$ & -0.31 & $9.08 \mathrm{e}-3$ & -0.37 & $1.67 \mathrm{e}-2$ & -0.11 \\
$3.91 \mathrm{e}-6$ & $3.50 \mathrm{e}-3$ & -0.89 & $2.77 \mathrm{e}-4$ & -0.87 & $1.28 \mathrm{e}-1$ & -0.39 & $1.18 \mathrm{e}-2$ & -0.38 & $1.93 \mathrm{e}-2$ & -0.21 \\
$1.95 \mathrm{e}-6$ & $6.67 \mathrm{e}-3$ & -0.93 & $5.23 \mathrm{e}-4$ & -0.91 & $1.78 \mathrm{e}-1$ & -0.47 & $1.60 \mathrm{e}-2$ & -0.43 & $2.46 \mathrm{e}-2$ & -0.35 \\
$9.77 \mathrm{e}-7$ & $1.28 \mathrm{e}-2$ & -0.94 & $9.98 \mathrm{e}-4$ & -0.93 & $2.73 \mathrm{e}-1$ & -0.62 & $2.35 \mathrm{e}-2$ & -0.56 & $3.42 \mathrm{e}-2$ & -0.48 \\
$4.88 \mathrm{e}-7$ & $2.43 \mathrm{e}-2$ & -0.93 & $1.90 \mathrm{e}-3$ & -0.93 & $4.60 \mathrm{e}-1$ & -0.75 & $3.81 \mathrm{e}-2$ & -0.70 & $5.04 \mathrm{e}-2$ & -0.56 \\
\hline
\end{tabular}

TABLE 2 Time step, error of velocity, velocity gradient and pressure with respective experimental order of convergence for the Stokes projection with grid parameter $h=0.05$

\begin{tabular}{lcccccccccc}
\hline$\Delta t$ & $\|U-u\|_{\ell^{\infty}\left(L^{2}\right)}$ & $\|U-u\|_{\ell^{2}\left(L^{2}\right)}$ & $\|\nabla(U-u)\|_{\ell^{\infty}\left(L^{2}\right)}$ & $\|\nabla(U-u)\|_{\ell^{2}\left(L^{2}\right)}$ & $\|P-p\|_{\ell^{2}\left(L^{2}\right)}$ \\
\hline $2.00 \mathrm{e}-3$ & $6.37 \mathrm{e}-4$ & - & $4.91 \mathrm{e}-5$ & - & $4.08 \mathrm{e}-2$ & - & $3.79 \mathrm{e}-3$ & - & $1.63 \mathrm{e}-2$ & - \\
$1.00 \mathrm{e}-3$ & $1.19 \mathrm{e}-3$ & -0.90 & $8.77 \mathrm{e}-5$ & -0.84 & $4.35 \mathrm{e}-2$ & -0.09 & $3.96 \mathrm{e}-3$ & -0.06 & $1.61 \mathrm{e}-2$ & 0.02 \\
$5.00 \mathrm{e}-4$ & $2.33 \mathrm{e}-3$ & -0.97 & $1.71 \mathrm{e}-4$ & -0.96 & $5.32 \mathrm{e}-2$ & -0.29 & $4.55 \mathrm{e}-3$ & -0.20 & $1.61 \mathrm{e}-2$ & -0.00 \\
$2.50 \mathrm{e}-4$ & $4.60 \mathrm{e}-3$ & -0.98 & $3.39 \mathrm{e}-4$ & -0.99 & $8.20 \mathrm{e}-2$ & -0.62 & $6.52 \mathrm{e}-3$ & -0.52 & $1.62 \mathrm{e}-2$ & -0.02 \\
$1.25 \mathrm{e}-4$ & $9.06 \mathrm{e}-3$ & -0.98 & $6.72 \mathrm{e}-4$ & -0.99 & $1.49 \mathrm{e}-1$ & -0.86 & $1.14 \mathrm{e}-2$ & -0.81 & $1.75 \mathrm{e}-2$ & -0.11 \\
$6.25 \mathrm{e}-5$ & $1.77 \mathrm{e}-2$ & -0.96 & $1.32 \mathrm{e}-3$ & -0.97 & $2.83 \mathrm{e}-1$ & -0.93 & $2.17 \mathrm{e}-2$ & -0.92 & $2.23 \mathrm{e}-2$ & -0.35 \\
$3.13 \mathrm{e}-5$ & $3.37 \mathrm{e}-2$ & -0.93 & $2.54 \mathrm{e}-3$ & -0.95 & $5.34 \mathrm{e}-1$ & -0.92 & $4.12 \mathrm{e}-2$ & -0.93 & $3.47 \mathrm{e}-2$ & -0.64 \\
$1.56 \mathrm{e}-5$ & $6.18 \mathrm{e}-2$ & -0.88 & $4.74 \mathrm{e}-3$ & -0.90 & $9.72 \mathrm{e}-1$ & -0.86 & $7.63 \mathrm{e}-2$ & -0.89 & $5.87 \mathrm{e}-2$ & -0.76 \\
$7.81 \mathrm{e}-6$ & $1.06 \mathrm{e}-1$ & -0.78 & $8.40 \mathrm{e}-3$ & -0.82 & $1.65 \mathrm{e}+0$ & -0.77 & $1.34 \mathrm{e}-1$ & -0.81 & $9.69 \mathrm{e}-2$ & -0.72 \\
$3.91 \mathrm{e}-6$ & $1.67 \mathrm{e}-1$ & -0.65 & $1.37 \mathrm{e}-2$ & -0.70 & $2.55 \mathrm{e}+0$ & -0.62 & $2.14 \mathrm{e}-1$ & -0.68 & $1.47 \mathrm{e}-1$ & -0.60 \\
$1.95 \mathrm{e}-6$ & $2.34 \mathrm{e}-1$ & -0.49 & $2.00 \mathrm{e}-2$ & -0.55 & $3.50 \mathrm{e}+0$ & -0.46 & $3.08 \mathrm{e}-1$ & -0.52 & $1.98 \mathrm{e}-1$ & -0.43 \\
$9.77 \mathrm{e}-7$ & $2.94 \mathrm{e}-1$ & -0.33 & $2.63 \mathrm{e}-2$ & -0.39 & $4.30 \mathrm{e}+0$ & -0.30 & $3.95 \mathrm{e}-1$ & -0.36 & $2.38 \mathrm{e}-1$ & -0.27 \\
$4.88 \mathrm{e}-7$ & $3.35 \mathrm{e}-1$ & -0.19 & $3.13 \mathrm{e}-2$ & -0.25 & $4.85 \mathrm{e}+0$ & -0.17 & $4.61 \mathrm{e}-1$ & -0.22 & $2.64 \mathrm{e}-1$ & -0.15 \\
\hline
\end{tabular}

Furthermore, we observe a smoothing of the error for $\Delta t \rightarrow 0$ for the Stokes projection. This seems not completely surprising, since we do not expect the discrete solution actually to tend to infinity. Note that also in the classical example in Dupont (1982), the error tends to a fixed value (the numerical solution there just decayed to 0 ) when $\Delta t \rightarrow 0$ and that our analysis provides just an upper bound for the error. The main point, however, is that for a large range of time step sizes the error increases and the negative order of convergence is close to the predicted one, at least for the velocity.

Thus, we may conclude that neither the Helmholtz nor the Stokes projection can cure the effect of too-frequent mesh changes.

In Fig. 7 we visualize profiles of the calculated solution at different times to illustrate how the solution deteriorates in time for a small time step size. 

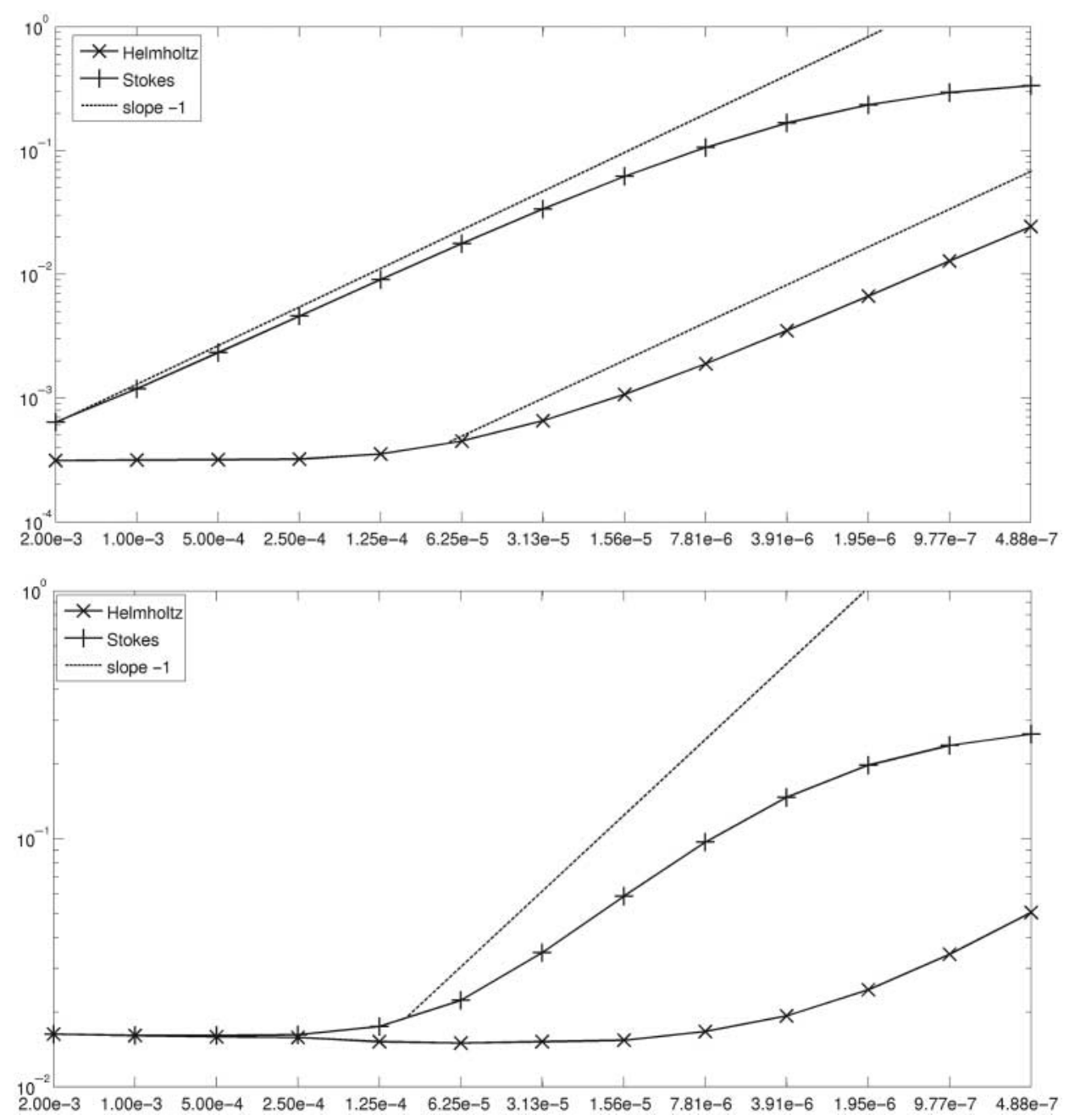

FIG. 6. Plots of velocity $\|U-u\|_{\ell^{\infty}\left(L^{2}\right)}$ and pressure error $\|P-p\|_{\ell^{2}\left(L^{2}\right)}$ versus time step size for grid parameter $h=0.05$.

EXAMPLE 5.2 In our second numerical experiment we study the model problem introduced in Besier \& Wollner (2011, Section 3.2). Here, the exact solution of the Stokes problem on $\Omega=(0,1)^{2}$ is given by

$$
\begin{aligned}
& u\left(x_{1}, x_{2}\right)=\left(\begin{array}{c}
\cos ^{2}\left(\frac{\pi x_{1}}{2}\right) \cos \left(\frac{\pi x_{2}}{2}\right) \sin \left(\frac{\pi x_{2}}{2}\right) \\
-\cos \left(\frac{\pi x_{1}}{2}\right) \sin \left(\frac{\pi x_{1}}{2}\right) \cos ^{2}\left(\frac{\pi x_{2}}{2}\right)
\end{array}\right), \\
& p\left(x_{1}, x_{2}\right)=\cos \left(\frac{\pi x_{1}}{2}\right) \sin \left(\frac{\pi x_{1}}{2}\right) \cos \left(\frac{\pi x_{2}}{2}\right) \sin \left(\frac{\pi x_{2}}{2}\right) .
\end{aligned}
$$



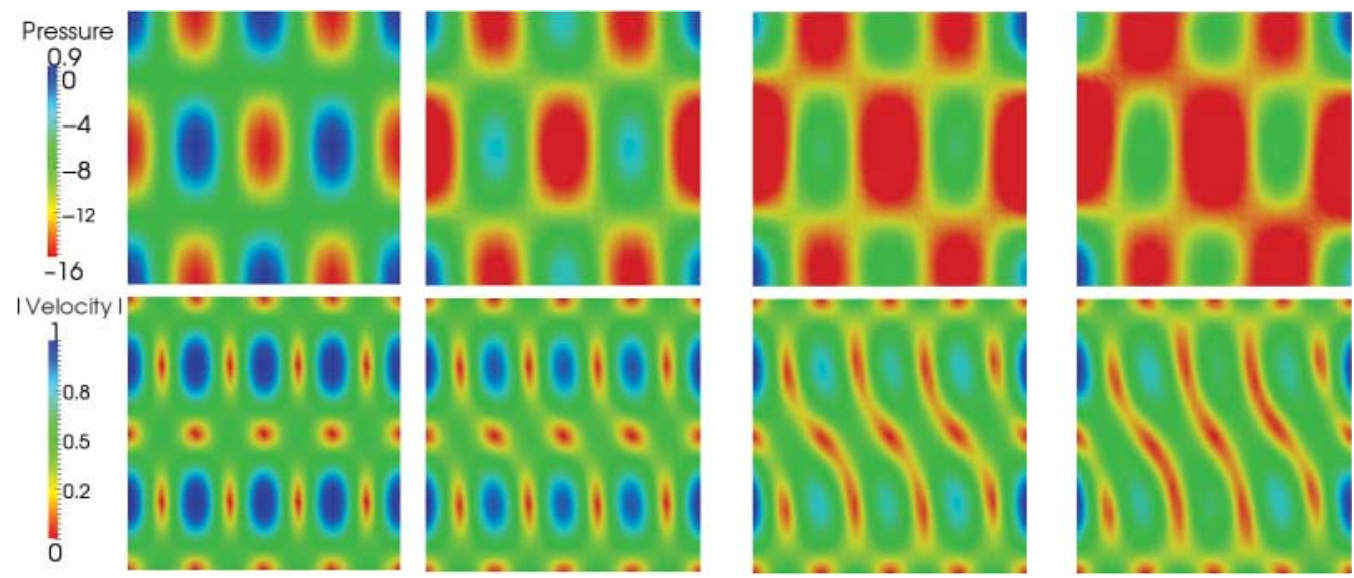

FIG. 7. Pressure (top) and velocity magnitude (bottom) for the Stokes projection with $\Delta t=3.91 \mathrm{e}-6$ and $h=0.05$ at times $t=0$, $6.25 \mathrm{e}-4,3.00 \mathrm{e}-3$ and $9.00 \mathrm{e}-3$. The figures show the deterioration of the solution in time for a small time step size.

TABLE 3 Time step, error of velocity and pressure with experimental order of convergence for Helmholtz and Stokes projection with grid parameter $h=0.05$

\begin{tabular}{|c|c|c|c|c|c|c|c|c|}
\hline \multirow{3}{*}{$\frac{\Delta t}{2.00 \mathrm{e}-3}$} & \multicolumn{4}{|c|}{ Helmholtz projection } & \multicolumn{4}{|c|}{ Stokes projection } \\
\hline & \multicolumn{2}{|c|}{$\|U-u\|_{L^{\infty}\left(\ell^{2}\right)}$} & \multicolumn{2}{|c|}{$\|P-p\|_{\ell^{2}\left(L^{2}\right)}$} & \multicolumn{2}{|c|}{$\|U-u\|_{\ell^{\infty}\left(L^{2}\right)}$} & \multicolumn{2}{|c|}{$\|P-p\|_{\ell^{2}\left(L^{2}\right)}$} \\
\hline & $2.79 \mathrm{e}-7$ & - & $6.54 \mathrm{e}-6$ & - & $3.16 \mathrm{e}-7$ & - & $6.53 e-6$ & - \\
\hline $1.00 \mathrm{e}-3$ & $2.75 e-7$ & 0.02 & $7.13 e-6$ & -0.13 & $.19 e-7$ & -0.41 & $.19 \mathrm{e}-6$ & -0.14 \\
\hline $5.00 \mathrm{e}-4$ & $2.75 e-7$ & -0.00 & $7.30 \mathrm{e}-6$ & -0.03 & $6.91 \mathrm{e}-7$ & -0.72 & $7.73 e-6$ & -0.10 \\
\hline $2.50 \mathrm{e}-4$ & $2.92 \mathrm{e}-7$ & -0.09 & $7.90 \mathrm{e}-6$ & -0.11 & $1.33 \mathrm{e}-6$ & -0.95 & $9.66 \mathrm{e}-6$ & -0.32 \\
\hline $1.25 \mathrm{e}-4$ & $3.59 \mathrm{e}-7$ & -0.30 & $9.62 \mathrm{e}-6$ & -0.28 & $2.73 e-6$ & -1.04 & $1.49 \mathrm{e}-5$ & -0.63 \\
\hline $6.25 e-5$ & $5.25 \mathrm{e}-7$ & -0.55 & $1.33 \mathrm{e}-5$ & -0.46 & $5.75 e-6$ & -1.07 & $2.62 \mathrm{e}-5$ & -0.81 \\
\hline $3.13 e-5$ & $8.10 \mathrm{e}-7$ & -0.63 & $1.90 \mathrm{e}-5$ & -0.52 & $1.23 e-5$ & -1.10 & $4.69 \mathrm{e}-5$ & -0.84 \\
\hline $1.56 \mathrm{e}-5$ & $1.26 \mathrm{e}-6$ & -0.63 & $2.64 \mathrm{e}-5$ & -0.48 & $2.64 \mathrm{e}-5$ & -1.10 & $8.18 \mathrm{e}-5$ & -0.80 \\
\hline $7.81 \mathrm{e}-6$ & $2.03 e-6$ & -0.69 & $3.58 \mathrm{e}-5$ & -0.44 & $5.56 \mathrm{e}-5$ & -1.08 & $1.36 \mathrm{e}-4$ & -0.73 \\
\hline $3.91 \mathrm{e}-6$ & $3.50 \mathrm{e}-6$ & -0.78 & $4.87 e-5$ & -0.44 & $1.13 \mathrm{e}-4$ & -1.02 & $2.12 \mathrm{e}-4$ & -0.64 \\
\hline $1.95 \mathrm{e}-6$ & $6.25 e-6$ & -0.84 & $6.74 \mathrm{e}-5$ & -0.47 & $2.17 \mathrm{e}-4$ & -0.94 & $3.09 \mathrm{e}-4$ & -0.54 \\
\hline
\end{tabular}

Again, in each time step, we switch the triangulation between the meshes shown in Fig. 5. The errors $\|U-u\|_{\ell^{\infty}\left(L^{2}\right)}$ and $\|P-p\|_{\ell^{2}\left(L^{2}\right)}$ for the Helmholtz and Stokes projection are given in Table 3 for grid parameter $h=0.05$ and visualized in Fig. 8.

In Besier \& Wollner (2011, Section 5.1), the authors analyse the behaviour of the pressure approximation for a decreasing sequence of time steps and a spatial mesh coarsened or refined in each time step. The Helmholtz and the Stokes projection are used for evaluating the velocity approximations on the new meshes. Their conclusion is that the divergence of the pressure variable can be avoided by using either projection. In contrast, our observation is that if the grid is changed in each time step in an 'uncontrolled' way, neither the Helmholtz nor the Stokes projection is capable of preventing a loss in the rate of convergence, or even the divergence, of the discrete approximations. Thus, this topic needs further investigation. 

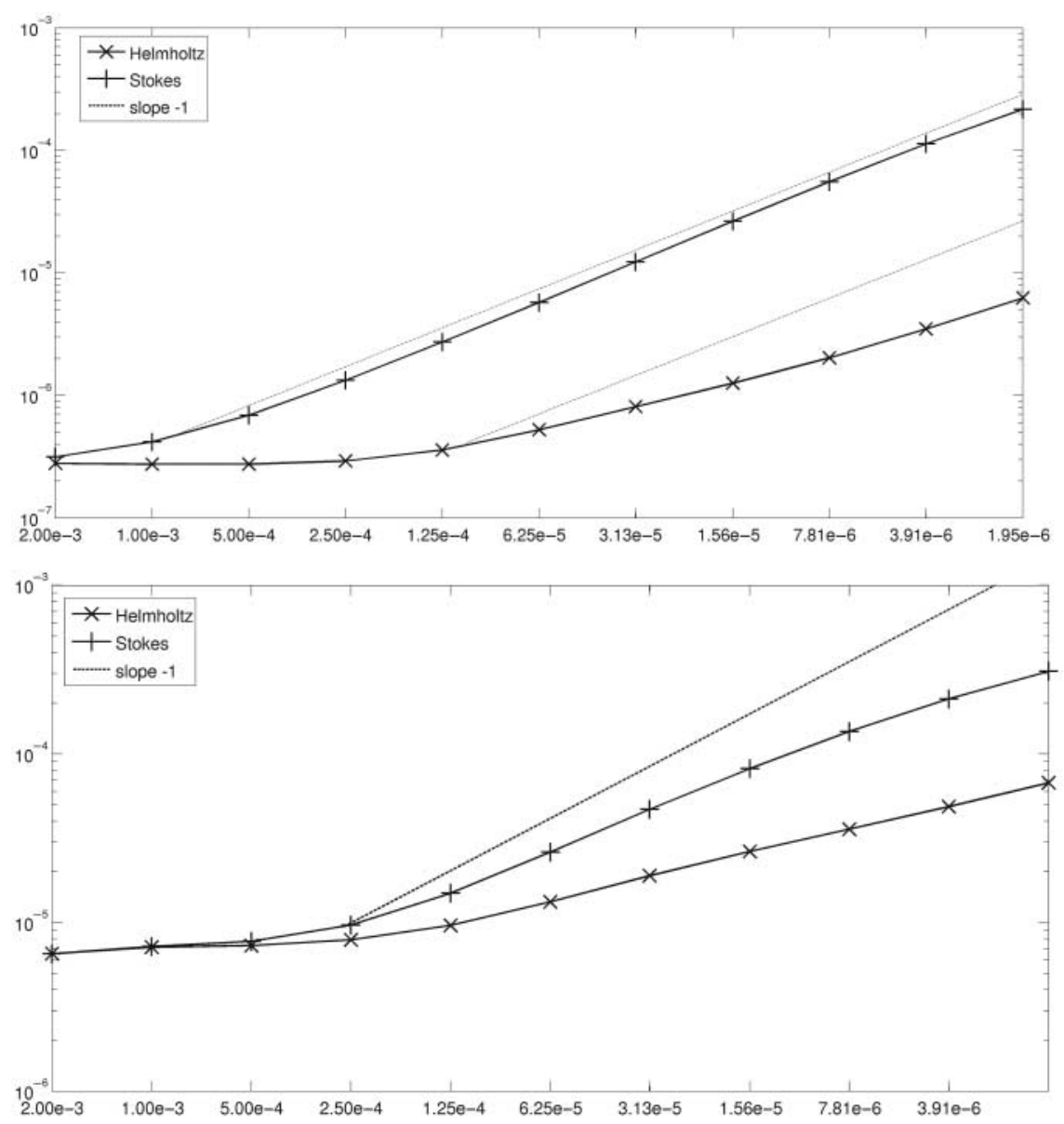

FIG. 8. Plots of velocity $\|U-u\|_{\ell^{\infty}\left(L^{2}\right)}$ and pressure error $\|P-p\|_{\ell^{2}\left(L^{2}\right)}$ versus time step size for grid parameter $h=0.05$.

REMARK 5.3 It is worthwhile mentioning that for the numerical simulations it was mandatory to compute the term on the right-hand side arising in Algorithm 2.2 given by $\left\langle\hat{U}_{n-1} / \Delta t_{n}, v_{n}\right\rangle$ exactly, that is, without performing variational crimes using numerical quadrature. If using numerical quadrature, the divergence of the solution would be much more pronounced and could even lead to blow-up in time of the error. This fact is somehow reminiscent of stability issues for the Lagrange-Galerkin method with nonexact integration; see Morton et al. (1988). For practical reasons we were therefore limited to mesh changes like those used in our experiments and we could not perform simulations for really arbitrary changes.

\section{Conclusion}

In this article we studied finite element approximations of the time-dependent Stokes system on dynamically changing meshes. Applying the most robust backward Euler method for time discretization we 
introduced the discrete Helmholtz or Stokes projection to evaluate the solution at time $t_{n}$ on the new mesh at time $t_{n+1}$. We proved the a priori estimates

$$
\begin{gathered}
\text { Helmholtz projection: } \quad\|U-u\|_{\ell^{\infty}\left(L^{2}\right)}=\mathcal{O}\left(\left\|U_{0}-u_{0}\right\|+\Delta t+h^{k+1}+\frac{h^{k+1}}{\Delta t}\right), \\
\text { Stokes projection: } \quad\|U-u\|_{\ell^{2}\left(L^{2}\right)}=\mathcal{O}\left(\left\|\nabla\left(U_{0}-u_{0}\right)\right\|+\Delta t+h^{k+1}+\frac{h^{k}}{\Delta t}\right),
\end{gathered}
$$

for the velocity error and

$$
\begin{array}{cl}
\text { Helmholtz projection: } & \|P-p\|_{\ell^{2}\left(L^{2}\right)}=\mathcal{O}\left(\left\|U_{0}-u_{0}\right\|+\Delta t+h^{k}+\frac{h^{k}}{\Delta t}\right), \\
\text { Stokes projection: } & \|P-p\|_{\ell^{2}\left(L^{2}\right)}=\mathcal{O}\left(\left\|\nabla\left(U_{0}-u_{0}\right)\right\|+\Delta t+h^{k}+\frac{h^{k}}{\Delta t}\right),
\end{array}
$$

for the pressure error. These surprisingly pessimistic upper bounds are illustrated by numerical examples giving evidence that the convergence rate is negative at least for a large range of time step sizes. These observations imply that using adaptive meshes for flow problems is delicate and needs further mathematical investigation.

\section{Funding}

\section{REFERENCES}

BäNSCH, E. (1998) Simulation of instationary, incompressible flows. Acta Math. Univ. Comenianae, 67, 101-114.

BäNSCH, E. (2001) Finite element discretization of the Navier-Stokes equations with a free capillary surface. Numer. Math., 88, 203-235.

BÄNSCh, E. \& SCHмidt, A. (2003) Simulation of dendritic crystal growth with thermal convection. Interfaces Free Bound., 2, 95-115.

BAuse, M. (2005) On optimum convergence rates for higher-order Navier-Stokes approximations. I. Error estimates for the spatial discretization. IMA J. Numer. Anal., 25, 812-841.

Besier, M. \& Wollner, W. (2011) On the pressure approximation in nonstationary incompressible flow simulations on dynamically varying spatial meshes. Int. J. Numer. Methods Fluids, 69, 1045-1064. doi:10.1002/fld.2625

Brezzi, F. \& Fortin, M. (1982) Mixed and Hybrid Finite Element Methods. Springer Series in Computational Mathematics. New York: Springer.

Cuvelier, C. (1985) A capillary free boundary problem governed by the Navier-Stokes equations. Comput. Methods Appl. Mech. Eng., 48, 45-80.

Dupont, T. (1982) Mesh modification for evolution equations. Math. Comp., 39, 85-107.

Eriksson, K. \& Johnson, C. (1991) Adaptive finite element methods for parabolic problems I: a linear model problem. SIAM J. Numer. Anal., 28, 43-77.

ERIKSSON, K. \& JoHNSON, C. (1995) Adaptive finite element methods for parabolic problems II: optimal error estimates in $L^{\infty}\left(L^{2}\right)$ and $L^{\infty}\left(L^{\infty}\right)$. SIAM J. Numer. Anal., 32, 706-740.

Girault, V. \& Raviart, P. A. (1987) Finite Element Method for Navier-Stokes Equations: Theory and Algorithms. Berlin: Springer. 
HE, Y. (2008) The Euler implicit/explicit scheme for the 2D time-dependent Navier-Stokes equations with smooth or non-smooth initial data. Math. Comp., 77, 2097-2124.

Heywood, J. G. \& RANNACHER, R. (1982) Finite-element approximation of the nonstationary Navier-Stokes problem. I. Regularity of solutions and second-order error estimates for spatial discretization. SIAM J. Numer. Anal., 19, 275-311.

Heywood, J. G. \& Rannacher, R. (1988) Finite-element approximation of the nonstationary Navier-Stokes problem III. Smoothing property and higher order error estimates for spatial discretization. SIAM J. Numer. Anal., 25, 489-512.

Heywood, J. G. \& Rannacher, R. (1990) Finite-element approximation of the nonstationary Navier-Stokes problem. Part IV: error analysis for second-order time discretization. SIAM J. Numer. Anal., 27, 353-384.

Hoffmann, J. \& Johnson, C. (2003) Adaptive Finite Element Methods for Incompressible Fluid Flow, vol. 25. Heidelberg: Springer.

KaraKashian, O. \& MAKridaKis, C. (2004) Convergence of a continuous Galerkin method with mesh modification for nonlinear wave equations. Math. Comp., 74, 85-102.

Morton, K. W., Priestly, A. \& SÜLI, E. (1988) Stability of the Lagrange-Galerkin method with non-exact integration. M2AN Math. Model. Numer. Anal., 22, 625-653.

Temam, R. (1983) Navier-Stokes Equations, Theory and Numerical Analysis, 3rd edn. Amsterdam: North-Holland.

Toвiska, L. \& Matthies, G. (2002) Finite element simulations of magnetic liquids with free boundary. Proc. Appl. Math. Mech., 1, 319-320.

YANG, D. (2000) Improved error estimation of dynamic finite element methods for second-order parabolic equations. J. Comput. Appl. Math., 126, 319-339. 